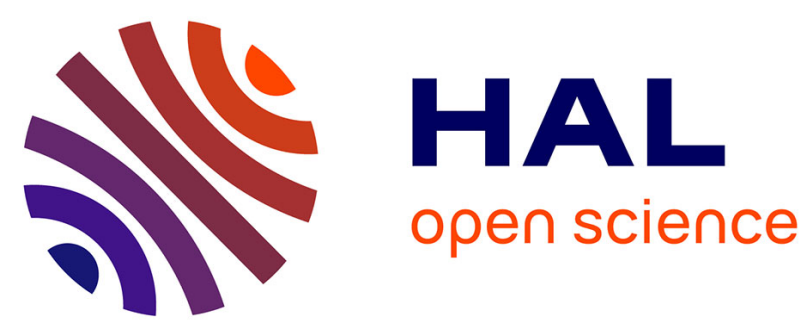

\title{
Bioinspired superoxide-dismutase mimics: The effects of functionalization with cationic polyarginine peptides
}

\author{
H.Y. Vincent Ching, Isabell Kenkel, Nicolas Delsuc, Emilie Mathieu, Ivana
}

Ivanović-Burmazović, Clotilde Policar

\section{- To cite this version:}

H.Y. Vincent Ching, Isabell Kenkel, Nicolas Delsuc, Emilie Mathieu, Ivana Ivanović-Burmazović, et al.. Bioinspired superoxide-dismutase mimics: The effects of functionalization with cationic polyarginine peptides. Journal of Inorganic Biochemistry, 2016, 160, pp.172-179. 10.1016/j.jinorgbio.2016.01.025 . hal-01288188

\section{HAL Id: hal-01288188 \\ https://hal.sorbonne-universite.fr/hal-01288188}

Submitted on 14 Mar 2016

HAL is a multi-disciplinary open access archive for the deposit and dissemination of scientific research documents, whether they are published or not. The documents may come from teaching and research institutions in France or abroad, or from public or private research centers.
L'archive ouverte pluridisciplinaire HAL, est destinée au dépôt et à la diffusion de documents scientifiques de niveau recherche, publiés ou non, émanant des établissements d'enseignement et de recherche français ou étrangers, des laboratoires publics ou privés. 


\section{Bioinspired superoxide-dismutase mimics: the effects of functionalization with cationic}

\section{polyarginine peptides}

H.Y. Vincent Ching, ${ }^{\text {a,b,c }}$ Isabell Kenkel, ${ }^{\mathrm{d}}$ Nicolas Delsuc, ${ }^{\mathrm{a}, \mathrm{b}, \mathrm{c}}$ Emilie Mathieu, ${ }^{\mathrm{a}, \mathrm{b}, \mathrm{c}}$ Ivana IvanovićBurmazovic, ${ }^{\mathrm{d}}$ and Clotilde Policar, ${ }^{\mathrm{a}, \mathrm{b}, \mathrm{c}} *$

a École Normale Supérieure - PSL Research University, Département de Chimie, 24 rue Lhomond, 75005 Paris, France

b Sorbonne Universités, UPMC Univ Paris 06, LBM, 4 place Jussieu, 75005, Paris, France

c CNRS, UMR 7203 Laboratoire des BioMolécules, LBM, 75005, Paris, France

d Department of Chemistry and Pharmacy, University of Erlangen-Nürnberg, Egerlandstr. 1, 91058

Erlangen, Germany

\section{Abstract}

Continuing a bio-mimetic approach, we have prepared peptide conjugates of a superoxide dismutase (SOD) mimic $[\mathbf{M n L}]^{+} \quad\left(\right.$ where $\mathbf{H L}=N$-(2-hydroxybenzyl)- $N, N^{\prime}$-bis[2-(Nmethylimidazolyl)methyl] ethane-1,2-diamine), namely [MnL'-Arg $\left.\mathbf{M}_{(n-1)}\right]^{n+}$ (where $n=2,4,7$ and 10) and $\left[\mathbf{M n L}^{\prime}-\mathbf{G l y}_{\mathbf{1}}\right]^{+} .\left[\mathbf{M n L}^{\prime}-\mathbf{A r g}_{(n-1)}\right]^{n+}$ contained cationic residue(s) that emulate the electrostatic channel of the enzyme. Physicochemical methods showed that functionalization at the secondary amine of $\mathbf{H L}$ did not impair coordination to $\mathrm{Mn}^{\mathrm{II}}$ with association constants $\left(K_{\text {assoc }}\right)$ between 1.6 to $3.3 \times 10^{6} \mathrm{M}^{-1}$. The $\mathrm{Mn}^{\mathrm{III}} / \mathrm{Mn}^{\mathrm{II}}$ redox potential of the conjugates were between 0.27 to $0.30 \mathrm{~V} v \mathrm{~s}$ SCE, slightly higher than $[\mathbf{M n L}]^{+}$under the same conditions, but remain at a value that facilitates $\mathrm{O}_{2}^{--}$dismutation. The catalytic rate constant $\left(k_{\mathrm{cat}}\right)$ of the dismutation for the series was studied using a direct stopped-flow method, which showed that for compounds with the same overall charge, the alkylation of the secondary amine of $[\mathrm{MnL}]^{+}\left(k_{\mathrm{cat}}=5.0 \pm 0.1 \times 10^{6} \mathrm{M}^{-1} \mathrm{~s}^{-1}\right)$ leaded to a lower value (i.e. for $\left[\mathbf{M n L}^{\prime}-\mathbf{G l y}_{1}\right]^{+}, k_{\mathrm{cat}}=4.2 \pm 0.1 \times 10^{6} \mathrm{M}^{-1} \mathrm{~s}^{-1}$ ). However, under the same conditions, $k_{\text {cat }}$ values between $5.0 \pm 0.4 \times 10^{6} \mathrm{M}^{-1} \mathrm{~s}^{-1}$ and $6.6 \pm 0.1 \times 10^{6} \mathrm{M}^{-1} \mathrm{~s}^{-1}$ were determined for $\left[\mathbf{M n L}^{\prime}-\mathbf{A r g}_{(n-1)}\right]^{n+}$ conjugates, indicating that the cationic residue(s) compensated for the lost in activity. Analysis of the effect of ionic strength on the $k_{\text {cat }}$ strongly suggested that not all the 
charges were involved, but only the closest ones electrostatically influenced the SOD active metal centre.

\section{Keywords}

Superoxide, SOD activity, SOD mimetics, SOD mimics, Manganese complexes, Peptide conjugate

\section{Introduction}

Superoxide $\left(\mathrm{O}_{2}{ }^{-}\right)$, a reactive oxygen species (ROS), is an endogenous byproduct of aerobic cellular respiration. Under normal conditions, the cellular concentration of $\mathrm{O}_{2}{ }^{--}$is regulated to the picomolar range by the superoxide dismutase (SOD) family of metalloenzymes that catalyze its dismutation $\left(2 \mathrm{O}_{2}^{--}+2 \mathrm{H}^{+} \rightarrow \mathrm{H}_{2} \mathrm{O}_{2}+\mathrm{O}_{2}\right)$ [1]. Three different types of SODs have been identified in mammals: SOD1, a CuZnSOD located in the cytosol, the nucleus, and the mitochondrial intermembrane space; SOD2, a MnSOD located in the mitochondrial matrix; and SOD3 (or EC-SOD), a CuZnSOD found in the extracellular plasma [2,3]. Oxidative stress occurs when SODs and other protective pathways against ROS are overwhelmed, and has been linked to various physiopathological processes including aging, cancer, inflammation, and neurodegenerative diseases [4-6]. As a result there has been great interest in developing therapeutic agents that mimic the activity of SOD to relieve the besieged protective pathways in pathological states. This research effort has often focused on synthetic Mn complexes as Mn ions do not induce Fenton chemistry, and their efficacy have been demonstrated both in vitro and in vivo [7-12].

The rate constant for the catalytic $\mathrm{O}_{2}{ }^{--}$dismutation $\left(k_{\text {cat }}\right)$ of SODs have been found to be close to the diffusion limit. This high efficiency has been attributed to physicochemical characteristics that are common to SODs across different lineage and these features can guide the design of SOD mimics [12]. For all known SODs, the metal redox potential (redox couple: $\mathrm{Cu}^{\mathrm{II}} / \mathrm{Cu}^{\mathrm{I}}, \mathrm{Mn}^{\mathrm{III}} / \mathrm{Mn}^{\mathrm{II}}$ and $\left.\mathrm{Fe}^{\mathrm{III}} / \mathrm{Fe}^{\mathrm{II}}\right)$ are close to $+0.12 \mathrm{~V}$ vs SCE, which is the mid-point between the 
$\mathrm{O}_{2}^{-}{ }^{-} / \mathrm{H}_{2} \mathrm{O}_{2}(+0.65 \mathrm{~V} v s \mathrm{SCE})$ and $\mathrm{O}_{2} / \mathrm{O}_{2}{ }^{--}(-0.40 \mathrm{~V} v s \mathrm{SCE})$ redox couples at $\mathrm{pH} 7$ [13]. At this midpoint, the kinetics are optimized for both the reduction and oxidation steps in the catalytic cycle [3,14]. However, it has been found that the metal active site represented less than $1 \%$ of the enzymes' surfaces [15-17]. In addition, the net charge of SODs is negative at physiological pH with isoelectric points ranging from 4.6 to 6.8 depending on the lineage $[16,17]$. Both features would be unfavourable for a reaction with the anionic substrate. However, a positively charged loop has been found in all known SODs, which contributes local electrostatic effects to guide $\mathrm{O}_{2}{ }^{-}$ from the protein exterior towards the metallic active site [3,17-19]. This is an important topographic feature compensating the small surface area of the active site and the overall negative charge of the proteins at physiological $\mathrm{pH}$ [12]. Indeed, the attraction of $\mathrm{O}_{2}{ }^{--}$by positive charges is a more general mechanism that has been shown to be also effective in low-molecular-weight molecules $[6,12,20-23]$.

Some of us have been developing a series of low-molecular-weight $\mathrm{Mn}^{\mathrm{III}} / \mathrm{Mn}^{\mathrm{II}}$ complexes, directly inspired by the active site of MnSOD, based on tertiary amine or 1,2-diamino-ethane, which reproduced the chemical environment of the Mn centre in SOD [24-30]. In native MnSOD, the $\mathrm{Mn}$ has been found to be coordinated in a trigonal bipyramidal geometry with an $\mathrm{N}_{3} \mathrm{O}$ /water coordination sphere containing a monodentate aspartate carboxylate and two histidine imidazoles ligated in the equatorial plane, and an imidazole from another histidine and an aqua/hydroxyl ligand occupying the apical positions [31]. By fine-tuning the nature of the Lewis bases (imidazole, carboxylate, pyridine or phenolate), we arrived at ( $N$-(2-oxybenzyl)- $N, N^{\prime}$-bis[2-( $N$ methylimidazolyl)methyl]-ethane-1,2-diamine)maganese(II) $\left([\mathbf{M n L}]^{+}\right)$[28]. This complex showed a $\mathrm{Mn}^{\mathrm{III}} / \mathrm{Mn}^{\mathrm{II}} E_{1 / 2}$ of $0.2 \mathrm{~V}$ vs SCE at $\mathrm{pH} 7.5$ (50 mM PIPES), which is close to the optimal value, an apparent kinetic constant $\left(k_{\mathrm{McCF}}\right.$ see a definition in [25] for instant) of $7.0 \pm 0.310^{6} \mathrm{M}^{-1} \mathrm{~s}^{-1}$, and in vitro anti-superoxide activity in activated RAW264.7 macrophages [28,32]. Continuing with this approach, we were interest in the influence of positively charged peptidic functionalization on the catalytic efficiency of MnSOD mimic. To the best of our knowledge, the only MnSOD mimic coupled to a peptide that has been reported in the literature is a Mn-porphyrinic SOD mimic conjugated to a mitochondrial signalling peptide [33]. This is surprising when considering the 
variety of metal complex-peptide conjugates that have been reported [34]. For the case of Mnporphyrinic SOD mimic, peptidic conjugation lead to an $80 \%$ decrease in the $k_{\text {cat }}$ value [33], however the source of this decrease in catalytic activity was not commented on. Herein we report the coupling of $[\mathbf{M n L}]^{+}$to cationic peptides including the cell penetrating peptide $\operatorname{Arg}_{9}$ [35] (scheme 1). We describe the influence of peptidic conjugation on the intrinsic properties of $[\mathrm{MnL}]^{+}$, such as the metal-ligand stability constant $\left(K_{\text {assoc }}\right)$ and electrochemical properties, as well as the consequences of these variations upon the $k_{\text {cat }}$ value and the involvement of second sphere electrostatic interactions.

\section{Experimental}

\subsection{Materials and methods}

Matrix-assisted laser desorption/ionization time of flight (MALDI-TOF) mass spectrometry analysis were performed in the ion positive reflector mode at the Plateforme de spectrométrie de masse et protéomique at the Université Pierre et Marie Curie on an ABI Voyager DE-Pro MALDITOF mass spectrometer (Applied Biosystems) using as matrix a saturated solution of a-cyano-4hydroxycinnamic acid in $\mathrm{ACN}: \mathrm{H}_{2} \mathrm{O}: \mathrm{TFA}$ (50:50:0.1). High resolution electrospray quadrupole coupled time of flight (HR-ESI-Q-TOF) mass spectrometry analysis were performed in the ion positive mode at the Institut de Chimie Moléculaire et des Matériaux d'Orsay at the Université Paris Sud, on a microTOF-Q II mass spectrometer (Bruker). Reversed phase HPLC was performed on a Waters 600 HPLC pump equipped with a Waters 2487 dual wavelength absorbance detector, using a XBridgeTM Prep C18 OBDTM column $(19$ x $50 \mathrm{~mm})$. The results were analysed using Waters Empower Pro® software. Analytical HPLC was performed on a Dionex Ultimate 3000 equipped with a variable wavelength detector, using an ACE 5 C18-300 column (250 x $4.6 \mathrm{~mm})$. The results were analysed using Chromeleon ${ }^{\circledR}$ software. Commercially available $\mathrm{D}_{2} \mathrm{O}$ of $99.5 \%$ isotopic purity was used for all NMR spectra. All NMR spectra were recorded at $300 \mathrm{~K}$ on a Bruker Ultrashield $300 \mathrm{MHz}$ AVANCE III spectrometer $\left({ }^{1} \mathrm{H}\right.$ at $300 \mathrm{MHz}$ and ${ }^{13} \mathrm{C}$ at $\left.76 \mathrm{MHz}\right)$. All 
NMR signals are reported in ppm. ${ }^{1} \mathrm{H}$ were referenced to the residue solvent peak. $1 \mathrm{D}{ }^{13} \mathrm{C}$ spectra were protons decoupled $\left(13 \mathrm{C}\left\{{ }^{1} \mathrm{H}\right\}\right)$. Coupling constants $(\boldsymbol{J})$ are reported in Hz. Peak multiplicities have been abbreviated as s (singlet), d (doublet), t (triplet), q (quartet), m (multiplet - unassignable multiplicity or overlapping signals), b (broad). Diastereotopic protons have been noted a and $b$, without any further attribution. MilliQ ${ }^{\mathrm{TM}}$ water was used for all experiments requiring water. All other solvents and reagents were purchase from commercial sources and used without further purification. HL was prepared as previously described [28].

\subsection{Synthesis}

Ligand synthesis: All functionalized ligands were prepared using standard solid phase peptide synthesis techniques. Examples are given for the synthesis of $\mathbf{H L}^{\prime}-\mathbf{A r g}_{\mathbf{1}}$ and $\mathbf{H L}^{\prime}-\mathbf{A r g}_{3}$.

\subsubsection{HL'-Arg 1 5TFA}

MBHA resin $(2.0 \mathrm{~g}, 1.0 \mathrm{mmol})$ was swollen in DCM. The solvent was removed and the yellow resin was washed with NMP (3 x $10 \mathrm{~mL})$. Boc-Arg(Tos)-OH (0.85 g $2.0 \mathrm{mmol}, 2$ eq.) and HBTU (0.76 g, 2.0 mmol, 2 eq.) were dissolved in NMP (10 mL). DIPEA (0.69 mL, 4.0 mmol, 4 eq.) was added and the solution was introduced to the resin. After shaking for $2 \mathrm{~h}$ the solution was removed and the resin washed with NMP $(3 \times 10 \mathrm{~mL})$ and DCM $(3 \times 10 \mathrm{~mL})$. TFA $(10 \mathrm{~mL})$ was added to the resin which turned red and was shaken for $5 \mathrm{~min}$. The deprotected loaded resin was neutralised with DiPEA/DCM (50/50 v/v) which returned to yellow and was washed with DCM (3 x $10 \mathrm{~mL}$ ). In the following order, DCM (10 mL), DIPEA (1.76 mL, $10.2 \mathrm{mmol}, 10$ eq.), and chloroacetyl chloride $(0.40 \mathrm{~mL}, 5.1 \mathrm{mmol}, 5$ eq. $)$ were added to the resin leading to a dark brown solution. After shaking for $2 \mathrm{~h}$ the solution was removed and the brown resin washed with DCM (3 x $10 \mathrm{~mL})$ and DMF (3 x $10 \mathrm{~mL})$. HL (1.1 g, $3.1 \mathrm{mmol}, 3$ eq.) was dissolved in DMF (10 mL). DIPEA (0.18 mL, $1.0 \mathrm{mmol}, 1$ eq.) was added and the solution was introduced to the resin. After shaking for $72 \mathrm{~h}$ the solution was removed and the resin washed with DMF (3 x $10 \mathrm{~mL}), \mathrm{MeOH}(3$ $\mathrm{x} 10 \mathrm{~mL})$ and dried in vacuo. Anisole $(4.0 \mathrm{~mL}), \mathrm{Me}_{2} \mathrm{~S}(0.67 \mathrm{~mL})$ and $\mathrm{HF}(15 \mathrm{~mL})$ were added to the resin. After stirring for $2 \mathrm{~h}$ at $0{ }^{\circ} \mathrm{C}$ the volatiles were removed in vacuo. The residue was triturated 
with cold diethyl ether and the mixture was filtered. To the residue, acetic acid/ $\mathrm{H}_{2} \mathrm{O}(2 \times 5 \mathrm{~mL}$, 50/50, v/v $)$ and $\mathrm{H}_{2} \mathrm{O}(2 \times 5 \mathrm{~mL})$ were added sequentially. The aqueous filtrates were combined and the solvents were removed in vacuo to give a yellow residue. Reverse-phase HPLC purification followed by lyophilisation afforded the pure product as a hydroscopic white solid $(0.10 \mathrm{~g}, 0.10$ mmol, $10 \%) .{ }^{1} \mathrm{H}$ NMR $\left(300 \mathrm{MHz}, \mathrm{D}_{2} \mathrm{O}\right): \delta=7.53\left(\mathrm{~d}, \boldsymbol{J}=2.0 \mathrm{~Hz}, 1 \mathrm{H}, \mathrm{H} 4_{\mathrm{im} 1}\right), 7.47(\mathrm{~d}, \boldsymbol{J}=2.0 \mathrm{~Hz}$, $\left.1 \mathrm{H}, \mathrm{H} 5_{\mathrm{im} 1}\right), 7.43\left(\mathrm{~m}, 2 \mathrm{H}, \mathrm{H} 4_{\mathrm{im} 2}\right.$ and $\left.\mathrm{H} 5_{\mathrm{im} 2}\right), 7.35\left(\mathrm{~m}, 2 \mathrm{H}, \mathrm{H} 4_{\phi}\right.$ and $\left.\mathrm{H} 6_{\phi}\right), 7.02(\mathrm{ddd}, \boldsymbol{J}=7.6 \mathrm{~Hz}, 7.6$ $\mathrm{Hz}$, and $\left.1.1 \mathrm{~Hz}, 1 \mathrm{H}, \mathrm{H} 5_{\phi}\right), 6.90\left(\mathrm{~d}, \boldsymbol{J}=8.1 \mathrm{~Hz}, 1 \mathrm{H}, \mathrm{H} 3_{\phi}\right), 4.87\left(\mathrm{~d}, \boldsymbol{J}=15.7 \mathrm{~Hz}, 1 \mathrm{H}, \mathrm{CH}_{2 \mathrm{a}(\mathrm{iml})}\right), 4.75$ $\left(\mathrm{d}, \boldsymbol{J}=15.7 \mathrm{~Hz}, 1 \mathrm{H}, \mathrm{CH}_{2 \mathrm{~b}(\mathrm{iml})}\right) 4.41\left(\mathrm{~d}, \boldsymbol{J}=15.7 \mathrm{~Hz}, 1 \mathrm{H}, \mathrm{CH}_{2 \mathrm{a}(\phi)}\right), 4.36\left(\mathrm{~d}, \boldsymbol{J}=15.7 \mathrm{~Hz}, 1 \mathrm{H}, \mathrm{CH}_{2 \mathrm{~b}(\phi)}\right)$, $4.25\left(\mathrm{dd}, \boldsymbol{J}=7.7 \mathrm{~Hz}\right.$ and $\left.5.9 \mathrm{~Hz}, 1 \mathrm{H}, \mathrm{H}^{\alpha}{ }_{\mathrm{Arg} 1}\right), 4.08\left(\mathrm{~s}, 2 \mathrm{H}, \mathrm{CH}_{2(\mathrm{im} 2)}\right), 3.85\left(\mathrm{~s}, 3 \mathrm{H}, \mathrm{CH}_{3(\mathrm{mml})}\right), 3.79(\mathrm{~s}$, $\left.3 \mathrm{H}, \mathrm{CH}_{3(\mathrm{im} 2)}\right), 3.63\left(\mathrm{~s}, 2 \mathrm{H}, \mathrm{H}^{\alpha}{ }_{\mathrm{Ac}}\right), 3.55\left(\mathrm{~m}, 2 \mathrm{H}, \mathrm{CH}_{2(\text { diamine })}\right), 3.25\left(\mathrm{~m}, 4 \mathrm{H}, \mathrm{H}_{\mathrm{Arg} 1}^{\delta}\right.$ and $\mathrm{CH}_{2 \text { (diamine2) })}$ ), $1.76\left(\mathrm{~m}, 4 \mathrm{H}, \mathrm{H}^{\beta}\right.$ Arg1 and $\left.\mathrm{H}_{\mathrm{Arg} 1}^{\gamma}\right) .{ }^{13} \mathrm{C}\left\{{ }^{1} \mathrm{H}\right\}$ NMR $\left(76 \mathrm{MHz}, \mathrm{D}_{2} \mathrm{O}\right): \delta=176.3\left(\mathrm{C}=\mathrm{O}_{\mathrm{Arg} 1}\right), 173.1$ $\left(\mathrm{C}=\mathrm{O}_{\mathrm{Ac}}\right), 162.9$ (TFA), $156.8\left(\mathrm{CH}_{5} \mathrm{~N}_{3(\mathrm{Arg} 1)}\right), 154.8\left(\mathrm{C}_{\phi}\right), 143.1\left(\mathrm{C} 2_{\mathrm{im} 2}\right), 137.1\left(\mathrm{C} 2_{\mathrm{im} 1}\right), 132.0\left(\mathrm{C6}_{\phi}\right)$, $131.7\left(\mathrm{C}_{\phi}\right), 125.3\left(\mathrm{C} 5_{\mathrm{im} 1}\right), 124.3\left(\mathrm{C} 5_{\mathrm{im} 2}\right), 121.0\left(\mathrm{C} 5_{\phi}\right), 120.3\left(\mathrm{C} 4_{\mathrm{im} 1}\right), 118.5\left(\mathrm{C} 4_{\mathrm{im} 2}\right), 116.8\left(\mathrm{C} 2_{\phi}\right)$, $115.4\left(\mathrm{C}_{\phi}\right), 56.0\left(\mathrm{C}^{\alpha}{ }_{\mathrm{Ac}}\right), 55.5\left(\mathrm{CH}_{2(\phi)}\right), 54.5\left(\mathrm{CH}_{2(\text { diamine })}\right), 54.0\left(\mathrm{C}^{\alpha}{ }_{\mathrm{Arg} 1}\right), 50.5\left(\mathrm{CH}_{2(\text { diamine } 2)}\right), 48.5$ $\left(\mathrm{CH}_{2(\mathrm{im} 2)}\right), 46.6\left(\mathrm{CH}_{2(\mathrm{im} 1)}\right), 40.6\left(\mathrm{C}^{\delta}{ }_{\mathrm{Arg} 1}\right), 34.9\left(\mathrm{CH}_{3(\mathrm{iml})}\right), 34.3\left(\mathrm{CH}_{3(\mathrm{im} 2)}\right), 28.1\left(\mathrm{C}^{\beta}{ }_{\mathrm{Arg} 1}\right), 24.6\left(\mathrm{C}_{\mathrm{Arg} 1}^{\gamma}\right)$. MALDI-TOF $(+): m / z \quad 462.3(100 \%) \quad\left[\mathrm{M}-5 \mathrm{TFA}-\left(\phi \mathrm{CH}_{2}\right)+2 \mathrm{H}^{+}\right]^{+}, 568.3(10 \%)\left[\mathrm{M}-5 \mathrm{TFA}+\mathrm{H}^{+}\right]^{+}$. HPLC: (C18A, 5-100\% ACN in $30 \mathrm{~min}) \mathrm{t}=7.92 \mathrm{~min}$, purity $99 \%$.

\subsection{2. $\mathrm{HL}^{\prime}-\mathrm{Arg}_{3}$ 7TFA}

Fmoc-Rink-Amid-MBHA resin $(0.48 \mathrm{~g}, 0.25 \mathrm{mmol})$, was swollen in DCM. The solvent was removed and the yellow resin was washed with NMP $(3 \times 5 \mathrm{~mL})$. Piperidine/NMP $(20 \% \mathrm{v} / \mathrm{v}, 5$ $\mathrm{mL})$ was added to the resin, shaken, and removed after $1 \mathrm{~min}$. A second portion $(5 \mathrm{~mL})$ was then added, shaken, and removed after $10 \mathrm{~min}$. The deprotected resin was then washed with NMP $(3 \times 5$ mL). Fmoc-Arg(Pbf)-OH (0.48 g 0.75 mmol, 3 eq. $)$ and HBTU ( 0.29 g, 0.75 mmol, 3 eq. $)$ were dissolved in NMP (5 mL). DIPEA (0.26 mL, $1.5 \mathrm{mmol}, 6$ eq.) was added and the solution was introduced to the resin. After shaking for $2 \mathrm{~h}$ the solution was removed and the resin washed with NMP (3 x $5 \mathrm{~mL})$. The deprotection and coupling steps were repeated two more times. In the 
following order, DCM (5 mL), DIPEA (0.43 mL, $2.5 \mathrm{mmol}, 10$ eq.), and chloroacetyl chloride $(0.10 \mathrm{~mL}, 1.3 \mathrm{mmol}, 5$ eq.) were added to the resin leading to a dark brown solution. After shaking for $2 \mathrm{~h}$ the solution was removed and the brown resin washed with DCM $(3 \times 5 \mathrm{~mL})$ and DMF $(3 \mathrm{x}$ $5 \mathrm{~mL})$. HL (0.27 g, $0.75 \mathrm{mmol}, 3$ eq.) was dissolved in DMF (5 mL). DIPEA (0.05 mL, 0.25 mmol, 1 eq.) was added and the solution was introduced to the resin. After shaking for $72 \mathrm{~h}$ the solution was removed and the resin washed with $\mathrm{DMF}(3 \times 5 \mathrm{~mL}), \mathrm{MeOH}(3 \times 5 \mathrm{~mL})$ and dried in vacuo. TFA/DCM $(5 \% \mathrm{v} / \mathrm{v}, 5 \mathrm{~mL})$ was added to the resin, shaken and the solution was collected after $1 \mathrm{~min}$. TFA/DCM (10\% v/v, $5 \mathrm{~mL})$ was added to resin, shaken and the solution was collected after $5 \mathrm{~min}$. TFA/TIS/ $\mathrm{H}_{2} \mathrm{O}(95: 2.5: 2.5 \mathrm{v} / \mathrm{v}, 5 \mathrm{~mL})$ was added, shaken and the solution was collected after $2 \mathrm{~h}$. The fractions were combined and the volatiles were removed in vacuo to give a yellow residue. Reverse-phase HPLC purification followed by lyophilisation afforded the pure product as a hydroscopic white solid $(0.025 \mathrm{~g}, 0.016 \mathrm{mmol}, 6 \%) .{ }^{1} \mathrm{H}$ NMR $\left(300 \mathrm{MHz}, \mathrm{D}_{2} \mathrm{O}\right): \delta=7.44(\mathrm{~d}, \boldsymbol{J}=$ $\left.2.0 \mathrm{~Hz}, 1 \mathrm{H}, \mathrm{H} 4_{\mathrm{im} 1}\right), 7.40\left(\mathrm{~m}, 3 \mathrm{H}, \mathrm{H} 5_{\mathrm{im} 1}, \mathrm{H} 4_{\mathrm{im} 2}\right.$ and $\left.\mathrm{H} 5_{\mathrm{im} 2}\right), 7.30\left(\mathrm{~m}, 2 \mathrm{H}, \mathrm{H} 4_{\phi}\right.$ and $\left.\mathrm{H} 6_{\phi}\right), 6.97$ (ddd, $\boldsymbol{J}$ $=7.6 \mathrm{~Hz}, 7.6 \mathrm{~Hz}$, and $\left.1.1 \mathrm{~Hz}, 1 \mathrm{H}, \mathrm{H} 5_{\phi}\right), 6.87\left(\mathrm{~d}, \boldsymbol{J}=8.1 \mathrm{~Hz}, 1 \mathrm{H}, \mathrm{H} 3_{\phi}\right), 4.59\left(\mathrm{~s}, 2 \mathrm{H}, \mathrm{CH}_{2(\mathrm{iml})}\right), 4.38$ (m, 3H, $\left.\mathrm{H}^{\alpha}{ }_{\mathrm{Arg} 1-3}\right), 4.24\left(\mathrm{~s}, 2 \mathrm{H}, \mathrm{CH}_{2(\phi)}\right), 4.07\left(\mathrm{~s}, 2 \mathrm{H}, \mathrm{CH}_{2(\mathrm{im} 2)}\right), 3.79\left(\mathrm{~s}, 3 \mathrm{H}, \mathrm{CH}_{3(\mathrm{iml})}\right), 3.77(\mathrm{~s}, 3 \mathrm{H}$, $\left.\mathrm{CH}_{3(\mathrm{im} 2)}\right), 3.56\left(\mathrm{~s}, 2 \mathrm{H}, \mathrm{H}^{\alpha}{ }_{\mathrm{Ac}}\right), 3.37\left(\mathrm{~m}, 2 \mathrm{H}, \mathrm{CH}_{2(\text { diamine } 1)}\right), 3.19\left(\mathrm{~m}, 8 \mathrm{H}, \mathrm{H}_{\text {Arg1-3 }}^{\delta}\right.$ and $\left.\mathrm{CH}_{2 \text { (diamine2) })}\right), 1.70$ $\left(\mathrm{m}, 12 \mathrm{H}, \mathrm{H}^{\beta}{ }_{\text {Arg1-3 }}\right.$ and $\left.\mathrm{H}_{\mathrm{Arg} 1-3}^{\gamma}\right) .{ }^{13} \mathrm{C}\left\{{ }^{1} \mathrm{H}\right\}$ NMR $\left(76 \mathrm{MHz}, \mathrm{D}_{2} \mathrm{O}\right): \delta=175.9\left(\mathrm{C}=\mathrm{O}_{\mathrm{Arg} 3}\right), 173.5$ $\left(\mathrm{C}=\mathrm{O}_{\mathrm{Arg} 1 / 2}\right), 173.4\left(\mathrm{C}=\mathrm{O}_{\mathrm{Arg} 1 / 2}\right), 172.6\left(\mathrm{C}=\mathrm{O}_{\mathrm{Ac}}\right), 162.9(\mathrm{TFA}), 156.8\left(\mathrm{CH}_{5} \mathrm{~N}_{3(\operatorname{Arg} 1-3)}\right), 154.7\left(\mathrm{Cl}_{\phi}\right)$, $143.3\left(\mathrm{C} 2_{\mathrm{im} 2}\right), 138.8\left(\mathrm{C} 2_{\mathrm{im} 1}\right), 131.5\left(\mathrm{C} 6_{\phi}\right), 131.4\left(\mathrm{C} 4_{\phi}\right), 124.9\left(\mathrm{C}_{\mathrm{im} 1}\right), 124.3\left(\mathrm{C} 5_{\mathrm{im} 2}\right), 120.9\left(\mathrm{C}_{\phi}\right)$, $120.0\left(\mathrm{C}_{\mathrm{im} 1}\right), 118.4\left(\mathrm{C} 4_{\mathrm{im} 2}\right), 116.6(\mathrm{TFA}), 115.5\left(\mathrm{C} 2_{\phi}\right), 114.5\left(\mathrm{C}_{\phi}\right), 56.1\left(\mathrm{C}^{\alpha}{ }_{\mathrm{Ac}}\right), 55.3\left(\mathrm{CH}_{2(\phi)}\right), 53.7$ $\left(\mathrm{CH}_{2(\text { diamine } 1)}\right), 53.5\left(\mathrm{C}^{\alpha}{ }_{\mathrm{Arg} 1 / 2}\right), 53.4\left(\mathrm{C}_{\mathrm{Arg} 1 / 2}^{\alpha}\right), 53.3\left(\mathrm{C}_{\mathrm{Arg} 3}^{\alpha}\right), 50.6\left(\mathrm{CH}_{2(\text { diamine2 })}\right), 48.2\left(\mathrm{CH}_{2(\mathrm{im} 2)}\right), 46.9$ $\left(\mathrm{CH}_{2(\mathrm{im} 1)}\right), 40.6\left(\mathrm{C}_{\mathrm{Arg} 1-3}^{\delta}\right), 34.6\left(\mathrm{CH}_{3(\mathrm{im} 1)}\right), 34.2\left(\mathrm{CH}_{3(\mathrm{im} 2)}\right), 28.5\left(\mathrm{C}^{\beta}{ }_{\mathrm{Arg} 3}\right), 28.2\left(\mathrm{C}_{\mathrm{Arg} 1,2}^{\beta}\right), 24.5\left(\mathrm{C}_{\mathrm{Arg} 1-3}^{\gamma}\right)$ MALDI-TOF (+): $m / z 774.6(100 \%) \quad\left[\mathrm{M}-7 \mathrm{TFA}-\left(\phi \mathrm{CH}_{2}\right)+2 \mathrm{H}^{+}\right]^{+}, 880.6(40 \%)\left[\mathrm{M}-7 \mathrm{TFA}+\mathrm{H}^{+}\right]^{+}$. HPLC: $(\mathrm{C} 18 \mathrm{~A}, 5-100 \% \mathrm{ACN}$ in $30 \mathrm{~min}) \mathrm{t}=8.48 \mathrm{~min}$, purity $99 \%$.

\subsubsection{HL'-Arg HOTFA $_{6}$}


Using MBHA resin $(0.41 \mathrm{~g}, 0.25 \mathrm{mmol})$, the produced was obtained as a white solid $(0.119$ g, $0.051 \mathrm{mmol}, 20 \%) .{ }^{1} \mathrm{H}$ NMR $\left(300 \mathrm{MHz}, \mathrm{D}_{2} \mathrm{O}\right): \delta=7.48\left(\mathrm{~d}, \boldsymbol{J}=2.0 \mathrm{~Hz}, 1 \mathrm{H}, \mathrm{H} 4_{\mathrm{im} 1}\right), 7.42(\mathrm{~d}, \boldsymbol{J}=$ $\left.2.0 \mathrm{~Hz}, 1 \mathrm{H}, \mathrm{H} 5_{\mathrm{im} 1}\right), 7.35$ (d, $\left.\boldsymbol{J}=2.0 \mathrm{~Hz}, 1 \mathrm{H}, \mathrm{H} 4_{\mathrm{im} 2}\right), 7.33\left(\mathrm{~d}, \boldsymbol{J}=2.0 \mathrm{~Hz}, 1 \mathrm{H}, \mathrm{H} 5_{\mathrm{im} 2}\right), 7.29(\mathrm{~m}, 2 \mathrm{H}$, $\mathrm{H}_{\phi}$ and $\left.\mathrm{H} 6_{\phi}\right), 6.93\left(\mathrm{ddd}, \boldsymbol{J}=7.6 \mathrm{~Hz}, 7.6 \mathrm{~Hz}\right.$, and $\left.1.1 \mathrm{~Hz}, 1 \mathrm{H}, \mathrm{H} 5_{\phi}\right), 6.84\left(\mathrm{~d}, \boldsymbol{J}=8.1 \mathrm{~Hz}, 1 \mathrm{H}, \mathrm{H} 3_{\phi}\right)$, $4.81\left(\mathrm{~m}, 2 \mathrm{H}, \mathrm{CH}_{2(\mathrm{~mm} 1)}\right), 4.40\left(\mathrm{~d}, \boldsymbol{J}=15.7 \mathrm{~Hz}, 1 \mathrm{H}, \mathrm{CH}_{2(\phi)}\right), 4.26\left(\mathrm{~m}, 6 \mathrm{H}, \mathrm{H}^{\alpha}{ }_{\text {Arg1-6 }}\right), 3.96(\mathrm{~m}, 2 \mathrm{H}$, $\left.\mathrm{CH}_{2(\mathrm{im} 2)}\right), 3.77\left(\mathrm{~s}, 3 \mathrm{H}, \mathrm{CH}_{3(\mathrm{~m} 1)}\right), 3.70\left(\mathrm{~s}, 3 \mathrm{H}, \mathrm{CH}_{3(\mathrm{im} 2)}\right), 3.55\left(\mathrm{~m}, 4 \mathrm{H}, \mathrm{H}^{\alpha}{ }_{\mathrm{Ac}}\right.$ and $\left.\mathrm{CH}_{2(\text { diamine })}\right), 3.25(\mathrm{~m}$, $14 \mathrm{H}, \mathrm{H}_{\text {Arg1-6 }}^{\delta}$ and $\left.\mathrm{CH}_{2 \text { (diamine2) })}\right), 1.67\left(\mathrm{~m}, 24 \mathrm{H}, \mathrm{H}^{\beta}{ }_{\text {Arg1-6 }}\right.$ and $\left.\mathrm{H}_{\mathrm{Arg} 1-6}^{\gamma}\right) .{ }^{13} \mathrm{C}\left\{{ }^{1} \mathrm{H}\right\} \mathrm{NMR}\left(765 \mathrm{MHz}, \mathrm{D}_{2} \mathrm{O}\right)$ : $\delta=175.9\left(\mathrm{C}=\mathrm{O}_{\mathrm{Arg}}\right), 173.5-173.1\left(\mathrm{C}=\mathrm{O}_{\mathrm{Arg} 1-5}\right), 172.4\left(\mathrm{C}=\mathrm{O}_{\mathrm{Ac}}\right), 162.8(\mathrm{TFA}), 156.7\left(\mathrm{CH}_{5} \mathrm{~N}_{3(\operatorname{Arg} 1-6)}\right)$, $154.8\left(\mathrm{C}_{\phi}\right), 142.8\left(\mathrm{C} 2_{\mathrm{im} 2}\right), 136.0\left(\mathrm{C} 2_{\mathrm{im} 1}\right), 132.3\left(\mathrm{C}_{\phi}\right), 131.6\left(\mathrm{C}_{\phi}\right), 125.5\left(\mathrm{C} 5_{\mathrm{im} 1}\right), 124.3\left(\mathrm{C} 5_{\mathrm{im} 2}\right)$, $121.0\left(\mathrm{C}_{\phi}\right), 120.5\left(\mathrm{C} 4_{\mathrm{im} 1}\right), 118.5\left(\mathrm{C} 4_{\mathrm{im} 2}\right), 116.3(\mathrm{TFA}), 115.8\left(\mathrm{C} 2_{\phi}\right), 115.4\left(\mathrm{C} 3_{\phi}\right), 55.5\left(\mathrm{C}^{\alpha}{ }_{\mathrm{Ac}}\right), 55.4$ $\left(\mathrm{CH}_{2(\phi)}\right), 54.05\left(\mathrm{CH}_{2 \text { (diamine })}\right), \quad 53.6-53.23\left(\mathrm{C}^{\alpha}{ }_{\text {Arg1- }}\right), 49.6\left(\mathrm{CH}_{2 \text { (diamine2) }}\right), 47.8\left(\mathrm{CH}_{2(\mathrm{im} 2)}\right), 46.2$ $\left(\mathrm{CH}_{2(\mathrm{iml} 1)}\right), 40.6\left(\mathrm{C}^{\delta}{ }_{\mathrm{Arg} 1-6}\right), 34.9\left(\mathrm{CH}_{3(\mathrm{iml} 1)}\right), 34.2\left(\mathrm{CH}_{3(\mathrm{~m} 2)}\right), 28.5-28.1\left(\mathrm{C}_{\mathrm{Arg} 1-6}^{\beta}\right), 24.4\left(\mathrm{C}_{\mathrm{Arg} 1-6}^{\gamma}\right)$. MALDI-TOF (+): $1242.8(100 \%)\left[\mathrm{M}-10 \mathrm{TFA}-\left(\phi \mathrm{CH}_{2}\right)+2 \mathrm{H}^{+}\right]^{+}, 1348.8(40 \%)\left[\mathrm{M}-10 \mathrm{TFA}+\mathrm{H}^{+}\right]^{+}$. HPLC: $(\mathrm{C} 18 \mathrm{~A}, 5-100 \% \mathrm{ACN}$ in $30 \mathrm{~min}) \mathrm{t}=8.81 \mathrm{~min}$, purity $98 \%$.

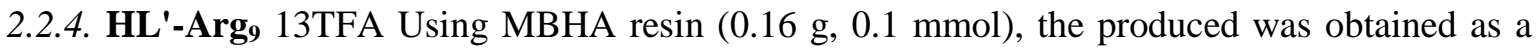
white solid (0.041 g, $0.013 \mathrm{mmol}, 13 \%) .{ }^{1} \mathrm{H}$ NMR $\left(300 \mathrm{MHz}, \mathrm{D}_{2} \mathrm{O}\right): \delta=7.40(\mathrm{~d}, \boldsymbol{J}=2.0 \mathrm{~Hz}, 1 \mathrm{H}$, $\left.\mathrm{H} 4_{\mathrm{im} 1}\right), 7.35\left(\mathrm{~m}, 3 \mathrm{H}, \mathrm{H} 5_{\mathrm{im} 1}, \mathrm{H} 4_{\mathrm{im} 2}, \mathrm{H} 5_{\mathrm{im} 2}\right), 7.26\left(\mathrm{~m}, 2 \mathrm{H}, \mathrm{H} 4_{\phi}\right.$ and $\left.\mathrm{H} 6_{\phi}\right), 6.92$ (ddd, $\boldsymbol{J}=7.6 \mathrm{~Hz}, 7.6$ $\mathrm{Hz}$, and $\left.1.1 \mathrm{~Hz}, 1 \mathrm{H}, \mathrm{H} 5_{\phi}\right), 6.82\left(\mathrm{~d}, \boldsymbol{J}=8.1 \mathrm{~Hz}, 1 \mathrm{H}, \mathrm{H} 3_{\phi}\right), 4.61\left(\mathrm{~s}, 2 \mathrm{H}, \mathrm{CH}_{2(\mathrm{ml})}\right), 4.28(\mathrm{~m}, 11 \mathrm{H}$, $\mathrm{H}^{\alpha}{ }_{\text {Arg1-9 }}$ and $\left.\mathrm{CH}_{2(\phi)}\right), 3.99\left(\mathrm{~s}, 2 \mathrm{H}, \mathrm{CH}_{2(\mathrm{im} 2)}\right), 3.74\left(\mathrm{~s}, 3 \mathrm{H}, \mathrm{CH}_{3(\mathrm{im} 1)}\right), 3.71\left(\mathrm{~s}, 3 \mathrm{H}, \mathrm{CH}_{3(\mathrm{im} 2)}\right), 3.50(\mathrm{~s}, 2 \mathrm{H}$, $\left.\mathrm{H}_{\text {Gly }}^{\alpha}\right), 3.37\left(\mathrm{~m}, 2 \mathrm{H}, \mathrm{CH}_{2 \text { (diamine })}\right), 3.15\left(\mathrm{~m}, 20 \mathrm{H}, \mathrm{H}_{\text {Arg1-9 }}^{\delta}\right.$ and $\left.\mathrm{CH}_{2 \text { (diamine2) })}\right), 1.67\left(\mathrm{~m}, 36 \mathrm{H}, \mathrm{H}^{\beta}{ }_{\text {Arg1-9 }}\right.$ and $\left.\mathrm{H}_{\mathrm{Arg} 1-9}^{\gamma}\right) .{ }^{13} \mathrm{C}\left\{{ }^{1} \mathrm{H}\right\}$ NMR $\left(76 \mathrm{MHz}, \mathrm{D}_{2} \mathrm{O}\right): \delta=175.9\left(\mathrm{C}=\mathrm{O}_{\text {Arg }}\right), 173.4-173.2\left(\mathrm{C}=\mathrm{O}_{\text {Arg1-8 }}\right), 172.4$ $\left(\mathrm{C}=\mathrm{O}_{\mathrm{Ac}}\right), 162.8(\mathrm{TFA}), 156.7\left(\mathrm{CH}_{5} \mathrm{~N}_{3(\mathrm{Arg} 1-9)}\right), 154.7\left(\mathrm{Cl}_{\phi}\right), 143.1\left(\mathrm{C} 2_{\mathrm{im} 2}\right), 138.0\left(\mathrm{C} 2_{\mathrm{im} 1}\right), 131.6\left(\mathrm{C6}_{\phi}\right)$, $131.5\left(\mathrm{C}_{\phi}\right), 125.0\left(\mathrm{C} 5_{\mathrm{im} 1}\right), 124.2\left(\mathrm{C}_{\mathrm{im}_{2}}\right), 120.8\left(\mathrm{C}_{\phi}\right), 120.1\left(\mathrm{C} 4_{\mathrm{im} 1}\right), 118.4\left(\mathrm{C}_{\mathrm{im} 2}\right), 116.3(\mathrm{TFA})$, $117.5\left(\mathrm{C} 2_{\phi}\right), 115.4\left(\mathrm{C} 3_{\phi}\right), 55.9\left(\mathrm{C}^{\alpha}{ }_{\mathrm{Ac}}\right), 55.4\left(\mathrm{CH}_{2(\phi)}\right), 53.6\left(\mathrm{CH}_{2(\text { diamine1 })}\right), 53.6-53.2\left(\mathrm{C}^{\alpha}{ }_{\text {Arg1-9 }}\right), 50.3$ $\left(\mathrm{CH}_{2(\text { diamine2 })}\right), 47.9\left(\mathrm{CH}_{2(\mathrm{im} 2)}\right), 46.7\left(\mathrm{CH}_{2(\mathrm{im} 1)}\right), 40.5\left(\mathrm{C}_{\mathrm{Arg} 1-9}^{\delta}\right), 34.6\left(\mathrm{CH}_{3(\mathrm{im} 1)}\right), 34.1\left(\mathrm{CH}_{3(\mathrm{im} 2)}\right), 28.4-$ 
$28.2\left(\mathrm{C}^{\beta}{ }_{\text {Arg1-9) }}\right), 24.3\left(\mathrm{C}^{\gamma}{ }_{\text {Argl }-9)}\right)$ MALDI-TOF (+): $1817.4(100 \%)\left[\mathrm{M}-13 \mathrm{TFA}+\mathrm{H}^{+}\right]^{+}, 1710.5(90 \%)$ [M-13TFA $\left.-\left(\phi \mathrm{CH}_{2}\right)+2 \mathrm{H}^{+}\right]^{+}$. HPLC: $(\mathrm{C} 18 \mathrm{~A}, 5-100 \% \mathrm{ACN}$ in $30 \mathrm{~min}) \mathrm{t}=9.21 \mathrm{~min}$, purity $99 \%$.

\subsubsection{HL'-Gly 1 4TFA}

Using Fmoc-Rink-Amid-MBHA resin $(0.75 \mathrm{~g}, 0.39 \mathrm{mmol})$, the produced was obtained as a white solid (0.085 g, $0.10 \mathrm{mmol}, 25 \%) .{ }^{1} \mathrm{H}$ NMR $\left(300 \mathrm{MHz}, \mathrm{D}_{2} \mathrm{O}\right): \delta=7.55(\mathrm{~d}, \boldsymbol{J}=2.0 \mathrm{~Hz}, 1 \mathrm{H}$, $\left.\mathrm{H} 4_{\mathrm{im} 1}\right), 7.48\left(\mathrm{~d}, \boldsymbol{J}=2.0 \mathrm{~Hz}, 1 \mathrm{H}, \mathrm{H} 5_{\mathrm{im} 1}\right), 7.41\left(\mathrm{~m}, 2 \mathrm{H}, \mathrm{H} 4_{\mathrm{im} 2}\right.$ and $\left.\mathrm{H} 5_{\mathrm{im} 2}\right), 7.35\left(\mathrm{~m}, 2 \mathrm{H}, \mathrm{H} 4_{\phi}\right.$ and $\left.\mathrm{H}_{\phi}\right)$, $7.02\left(\mathrm{ddd}, \boldsymbol{J}=7.6 \mathrm{~Hz}, 7.6 \mathrm{~Hz}\right.$, and $\left.1.1 \mathrm{~Hz}, 1 \mathrm{H}, \mathrm{H} 5_{\phi}\right), 6.88\left(\mathrm{~d}, \boldsymbol{J}=8.1 \mathrm{~Hz}, 1 \mathrm{H}, \mathrm{H} 3_{\phi}\right), 4.85(\mathrm{~s}, 2 \mathrm{H}$, $\left.\mathrm{CH}_{2(\mathrm{iml})}\right), 4.44\left(\mathrm{~s}, 2 \mathrm{H}, \mathrm{CH}_{2(\phi)}\right), 4.05\left(\mathrm{~s}, 2 \mathrm{H}, \mathrm{CH}_{2(\mathrm{im} 2)}\right), 3.95\left(\mathrm{~s}, 2 \mathrm{H}, \mathrm{H}^{\alpha}{ }_{\mathrm{Gly}}\right), 3.85\left(\mathrm{~s}, 3 \mathrm{H}, \mathrm{CH}_{3(\mathrm{iml})}\right), 3.76$ (s, $\left.3 \mathrm{H}, \mathrm{CH}_{3(\mathrm{~m} 2)}\right), 3.62\left(\mathrm{~s}, 2 \mathrm{H}, \mathrm{H}^{\alpha}{ }_{\mathrm{Ac}}\right), 3.60\left(\mathrm{t}, \boldsymbol{J}=5.7 \mathrm{~Hz}, 2 \mathrm{H}, \mathrm{CH}_{2 \text { (diamine }) \text { ) }}, 3.28(\mathrm{t}, \boldsymbol{J}=5.7 \mathrm{~Hz}, 2 \mathrm{H}\right.$, $\left.\mathrm{CH}_{2 \text { (diamine2) })}\right){ }^{13} \mathrm{C}\left\{{ }^{1} \mathrm{H}\right\}$ NMR $\left(76 \mathrm{MHz}, \mathrm{D}_{2} \mathrm{O}\right): \delta=173.7\left(\mathrm{C}=\mathrm{O}_{\mathrm{Gly} 1}\right.$ and $\left.\mathrm{C}=\mathrm{O}_{\mathrm{Ac}}\right), 154.8\left(\mathrm{C}_{\phi}\right), 143.0$ $\left(\mathrm{C} 2_{\mathrm{im} 2}\right), 136.0\left(\mathrm{C} 2_{\mathrm{im} 1}\right), 132.3\left(\mathrm{C}_{\phi}\right), 131.8\left(\mathrm{C} 4_{\phi}\right), 125.5\left(\mathrm{C} 5_{\mathrm{im} 1}\right), 124.3\left(\mathrm{C5}_{\mathrm{im} 2}\right), 121.0\left(\mathrm{C} 5_{\phi}\right), 120.5$ $\left(\mathrm{C} 4_{\text {im } 1}\right), 118.5\left(\mathrm{C}_{\mathrm{im}_{2}}\right), 116.0\left(\mathrm{C} 2_{\phi}\right), 115.4\left(\mathrm{C}_{\phi}\right), 55.9\left(\mathrm{C}^{\alpha}{ }_{\mathrm{Ac}}\right), 55.6\left(\mathrm{CH}_{2(\phi)}\right), 54.7\left(\mathrm{CH}_{2(\text { diamine } 1)}\right), 50.2$ $\left(\mathrm{CH}_{2 \text { (diamine2) })}\right), 48.5\left(\mathrm{CH}_{2(\mathrm{im} 2)}\right), 46.3\left(\mathrm{CH}_{2(\mathrm{im} 1)}\right), 42.0\left(\left(\mathrm{C}_{\mathrm{Gly}}^{\alpha}\right), 35.0-\left(\mathrm{C}^{\beta}{ }_{\mathrm{Tyr} 1}\right), 34.5\left(\mathrm{CH}_{3(\mathrm{im} 1)}\right), 34.2\right.$ $\left(\mathrm{CH}_{3(\mathrm{im} 2)}\right)$ MALDI-TOF (+): $469.6(100 \%)$ [M-4TFA+H $\left.{ }^{+}\right]^{+}$. HPLC: $(\mathrm{C} 18 \mathrm{~A}, 5-100 \%$ ACN in 30 $\min ) \mathrm{t}=7.61 \mathrm{~min}$, purity $96 \%$.

\section{3. in situ complex formation}

Unless otherwise stated, aqueous solutions of $\left[\mathbf{M n L} \mathbf{L}^{\prime}-\mathbf{R}\right]^{n+}$ were prepared by mixing stoichiometric amounts of $\mathrm{MnCl}_{2}$ and $\mathbf{H L}$ '- $\mathbf{R}$ in aqueous ammonium acetate (100 mM, pH 8) for mass spectrometry, and HEPES (100 mM, pH 7.5) for other studies.

$\left[\text { MnL'-Arg }_{1}\right]^{2+}:$ HR-ESI-Q-TOF (+) Calcd for $[\mathrm{M}]^{2+}, \mathrm{C}_{27} \mathrm{H}_{41} \mathrm{MnN}_{11} \mathrm{O}_{3}: \mathrm{m} / \mathrm{z} 311.1382$. Found $\mathrm{m} / \mathrm{z}$ 311.1385; Calcd for $\left[\mathrm{M}-\mathrm{H}^{+}\right]^{+}, \mathrm{C}_{27} \mathrm{H}_{40} \mathrm{MnN}_{11} \mathrm{O}_{3}: \mathrm{m} / \mathrm{z}$ 621.2691. Found $\mathrm{m} / \mathrm{z}$ 621.2678.

$\left[\mathbf{M n L}^{\prime}-\mathrm{Arg}_{3}\right]^{4+}:$ HR-ESI-Q-TOF (+) Calcd for $\left[\mathrm{M}-\mathrm{H}^{+}\right]^{3+}, \mathrm{C}_{39} \mathrm{H}_{66} \mathrm{MnN}_{19} \mathrm{O}_{5}: \mathrm{m} / \mathrm{z} 311.8286$. Found m/z 311.8298; Calcd for $\left[\mathrm{M}-2 \mathrm{H}^{+}\right]^{2+}, \mathrm{C}_{39} \mathrm{H}_{65} \mathrm{MnN}_{19} \mathrm{O}_{5}: \mathrm{m} / \mathrm{z}$ 467.2393. Found m/z 467.2387. 
$\left[\mathrm{MnL}^{\prime}-\mathrm{Arg}_{6}\right]^{7+}:$ HR-ESI-Q-TOF $(+)$ Calcd for $\left[\mathrm{M}-5 \mathrm{H}^{+}\right]^{2+}, \quad \mathrm{C}_{57} \mathrm{H}_{101} \mathrm{MnN}_{31} \mathrm{O}_{8}: \quad \mathrm{m} / \mathrm{z}$ 701.3909. Found $\mathrm{m} / \mathrm{z} 701.3884$.

$\left[\text { MnL'-Gly }_{1}\right]^{+}$: HR-ESI-Q-TOF (+) Calcd for $[\mathrm{M}]^{+}, \mathrm{C}_{23} \mathrm{H}_{31} \mathrm{MnN}_{8} \mathrm{O}_{3}: \mathrm{m} / \mathrm{z}$ 522.1894. Found $\mathrm{m} / \mathrm{z} 522.1884$.

\subsection{UV-vis spectrophotometric titrations}

The UV visible spectra of buffered aqueous solution (HEPES, $100 \mathrm{mM}, \mathrm{pH}$ 7.5) of $\mathbf{H L}$ '-R $(\sim 500 \mu \mathrm{M})$ in the presence of $\mathrm{MnCl}_{2}(0$ to $\sim 800 \mu \mathrm{M})$ were recorded on a CARY-5 UV-vis spectrophotometer in stoppered semi-micro quartz cuvettes $(1500 \mu \mathrm{L}, 1 \mathrm{~cm}$ path length). The data were collected between 250 and $350 \mathrm{~nm}$ at $25^{\circ} \mathrm{C}$.

\subsection{Isothermal titration calorimetry}

ITC were performed using a TA instrument Nano-ITC calorimeter operating with a reference power of $166 \mu \mathrm{J} / \mathrm{s}$, and a stirring speed of $1000 \mathrm{rpm}$. A buffered aqueous solution (HEPES, $100 \mathrm{mM}, \mathrm{pH} 7.5)$ of $\mathrm{MnCl}_{2}(\sim 8 \mathrm{mM}, 200 \mu \mathrm{L})$ after an initial 'dummy' injection $(2 \mu \mathrm{L}$ with $300 \mathrm{~s}$ interval) was titrated in 25 injections (10 $\mu \mathrm{L}$ with $300 \mathrm{~s}$ intervals) into a buffered aqueous solutions (HEPES, $100 \mathrm{mM}, \mathrm{pH} 7.5)$ of $\mathbf{H L}-\mathbf{R}(\sim 0.7 \mathrm{mM}, 983 \mu \mathrm{L})$. Triplicate titrations were performed. The data were processed using the inbuilt software. For the integrated heat data, a zero-order polynomial baseline was taken using the average of the last five data points.

\subsection{Cyclic voltammetry}

Cyclic voltammograms were recorded at room temperature on a Metrohm potentiostat (AUTOLAB model). The auxiliary electrode was a Pt wire and the working electrode was a glassy carbon disk carefully polished before each voltammogram with a $1 \mu \mathrm{m}$ diamond paste, sonicated in an ethanol bath, washed with ethanol and finally air dried. The reference electrode was $\mathrm{Ag} / \mathrm{AgCl}$ which was calibrated against SCE before use. The ohmic drop was systematically compensated using the adequate option within the commercial potentiostat. 


\subsection{Determination of in vitro SOD activity via stopped-flow technique}

Superoxide dismutase activity was tested by a direct method using stopped-flow technique as described elsewhere [36]. Experiments were carried out on a Biologic SFM-400 instrument, using syringes 1, 2 and 3, combined with a Energetiq LDLS ENQ EQ-99-FC laser driven light source and a J\&M TIDAS diode array detector (integration time $0.5 \mathrm{~ms}, \lambda=180-724 \mathrm{~nm}$ ). The source of $\mathrm{O}_{2}{ }^{-}$was commercially available $\mathrm{KO}_{2}$ dissolved in dry DMSO $\left(\left[\mathrm{O}_{2}{ }^{-}\right] \approx 1-2 \mathrm{mM}\right)$. Complexes were used in at least four different concentrations (between 0.9 and $15 \mu \mathrm{M}$ in $60 \mathrm{mM}$ HEPES buffer, $\mathrm{pH}=7.4$ ). Millipore water was used for the preparation of the buffer solutions, the ionic strength $(\mu)$ was adjusted by addition of $0,86,186$ and $286 \mathrm{mM} \mathrm{NaCl}$. Buffers were treated at least $12 \mathrm{~h}$ with Chelex 100 sodium exchange resin before use. In the stopped-flow experiment aqueous complex solution was mixed in a 10:1 ratio with the superoxide solution in DMSO by the use of a high-density mixer. $\mathrm{O}_{2}{ }^{-}$concentration exceeded complex concentration in at least ten fold excess to ensure catalytic conditions. Data analysis was performed using BioKine V4.66 software. Each $k_{\text {obs }}$ value is a mean value of at least 10 values. $k_{\text {cat }}$ values were determined from the slope of the $k_{\text {obs }}$ vs. [MnL'] plot.

\section{Results and Discussion}

\subsection{Ligand synthesis}

The synthesis of peptidic derivatives $(\mathbf{H L}$ '-R) of the parent ligand $(\mathbf{H L})$ was performed using standard solid phase peptide synthesis techniques. Briefly, for each peptide, protected amino acids were coupled sequentially followed by a short chloroacetyl linker at the $\mathrm{N}$-terminus via the Schotten-Baumann reaction. Nucleophilic substitution of the halide by the secondary amine of $\mathbf{H L}$ followed by deprotection of amino acids side chains and cleavage gave the desired product which was purified by preparative reversed-phase HPLC, and fully characterised by multinuclear 1- and 2D NMR spectroscopy, and positive-mode MALDI-TOF mass spectrometry. >95\% purity, determined by analytical reversed-phase HPLC, was obtained for each product. 


\subsection{Complexation}

The UV-vis spectrum of each HL'-R in aqueous HEPES buffer (100 mM pH 7.5) exhibited an absorption band at $\lambda_{\max } \approx 273 \mathrm{~nm}$ corresponding to the $\pi$ - $\pi^{*}$ transition of the phenol moiety. As $\mathrm{Mn}^{\mathrm{II}}$ ion was titrated into the system, the intensity of this band decreased while a new absorption band at $\lambda_{\max } \approx 286 \mathrm{~nm}$ appeared with isobestic points at ca. 262 and $276 \mathrm{~nm}$ (e.g. Fig. 1). The new band was assigned to the $\pi$ - $\pi^{*}$ transition of the phenolate group, consistent with the coordination of the $\mathbf{L}^{\prime}-\mathbf{R}$ to $\mathrm{Mn}^{\mathrm{II}}$ ion [28]. There were no further spectral changes after reaching one equivalent of $\mathrm{Mn}^{\mathrm{II}}$ ion confirming the 1:1 metal-to-ligand stoichiometry $(N)$. The complexation was also supported by positive mode HR-ESI-Q-TOF mass spectrometry where molecular ions corresponding to $\left[\mathbf{M n L}^{\prime}-\mathbf{A r g}_{1}\right]^{+},\left[\mathbf{M n L}^{\prime}-\mathbf{A r g}_{3}\right]^{4+}$ and $\left[\mathbf{M n L} \mathbf{L}^{\prime}-\mathbf{A r g}_{6}\right]^{7+}$ in different protonation states, and $\left[\mathbf{M n L} \mathbf{L}^{\prime}-\mathbf{G}_{\mathbf{1}}\right]^{+}$were observed. However, molecular ions arising from $\left[\mathbf{M n L} \mathbf{L}^{\prime}-\mathbf{A r g}_{\mathbf{9}}\right]^{\mathbf{1 0 +}}$ were not detected under the mass spectrometry conditions, and this may be due to the high charge of the molecular ions.

Isothermal titration calorimetry (ITC) was used to determine the thermodynamic parameters of $\left[\mathbf{M n L} \mathbf{L}^{\prime}-\mathbf{R}\right]^{n+}$ formation (table 1). The experimental ITC titration curves (e.g. Fig. 1) were readily fitted to a one-site binding model with $N$ values close to 1 and $K_{\text {assoc }}$ values in the range of $10^{6} \mathrm{M}^{-1}$ which were comparable or of higher affinity than the parent compound. In all cases, the complexation reaction had a large and favourable entropic component with the $-\mathrm{T} \Delta S / \Delta G$ ratio ranging from 1.3 to 1.6 and an unfavourable enthalpic component with the $\Delta H / \Delta G$ ratio ranging from -0.3 to -0.6 . Entropically dominated chelation have been previously reported for other 1:1 $\mathrm{Mn}^{\mathrm{II}}$ to ligand complexes and was attributed to a significant contribution from the desolvation of the reactants [37-39]. Direct comparison of $[\mathbf{M n L}]^{+}$with $[\mathbf{M n L}-\mathbf{G l y}]^{+}$revealed that alkylation of the HL secondary amine enhanced the $K_{\text {assoc }}$ value twofold. This was unexpected since tertiary amines have frequently been found to be less donating, due to delocalization and also to increase bulkiness. This can be seen here with $\Delta H$ being more positive (less favourable) for the conjugate ligand. In the series, $\Delta G$ became slightly more negative (more favourable) due to an increase in $\Delta S$. For the polyarginine derivatives $\left(\left[\mathbf{M n L} \mathbf{L}^{\prime}-\mathbf{A r g}_{1}\right]^{2+}\right.$ to $\left.\left[\mathbf{M n L}^{\prime}-\mathbf{A r g}_{\mathbf{9}}\right]^{10+}\right)$, the $K_{\text {assoc }}$ value decreased 
(3.3 to $1.6 \times 10^{6} \mathrm{M}^{-1}$ ) with increasing increments of positively charged residues, which could be ascribed to the increasing electrostatic repulsion between the positively charged $\mathrm{Mn}^{\mathrm{II}}$ ion and ligand. With the exception of $\left[\mathbf{M n L}^{\prime}-\mathbf{A r g}_{9}\right]^{10+}$, this electrostatic effect is characterised by the $\Delta H$ value becoming more positive as the polyarginine lengthens. In the case of $\left[\mathbf{M n L} \mathbf{L}^{\prime}-\mathbf{A r g}_{9}\right]^{10+}$, it is possible that the $\Delta H$ value does not follow this trend because of secondary structural effects, which became more prominent in the longer peptide, contributing to both entropic and enthalpic components.

\subsection{Electrochemistry}

$1.0 \mathrm{mM}$ aqueous solutions of $\left[\mathbf{M n L} \mathbf{L}^{\prime}-\mathbf{R}\right]^{n+}$ were prepared in situ and studied by cyclic voltammetry. For the parent compound, $[\mathrm{MnL}]^{+}$, the $E_{1 / 2}$ value was $0.22 \mathrm{~V}$ vs $\mathrm{SCE}$, which was comparable to those values previously reported in different aqueous buffers. At slow scan rate $(v=$ $0.05 \mathrm{~V} / \mathrm{s}$ ), the cyclic voltammogram (CV) of $[\mathbf{M n L} \text {-Gly }]^{+}$(Fig. 2 left) exhibit an anodic wave at $E_{\mathrm{p}}{ }^{\mathrm{a}}=0.34 \mathrm{~V}$ vs SCE (wave $a$ ) and two cathodic waves on the reverse scan at $E_{\mathrm{p}}{ }^{\mathrm{c}}=0.25$ and $0.10 \mathrm{~V}$ vs. SCE (wave $b$ and $b^{\prime}$ ). However, as the scan rate increased only one cathodic wave was observed on the reverse scan, for example at $v=0.50 \mathrm{~V} / \mathrm{s},{E_{\mathrm{p}}}^{\mathrm{a}}=0.36 \mathrm{~V}$ vs SCE and $E_{\mathrm{p}}{ }^{\mathrm{c}}=0.24 \mathrm{~V}$ vs SCE, with $E_{1 / 2}=0.30 \mathrm{~V}$ vs SCE. Fig. 3 left shows CV of $\left[\mathbf{M n L}^{\prime}-\mathbf{G l y}_{1}\right]^{+}$collected between pH 5.6 and 8 at slow scan rate $(v=0.10 \mathrm{~V} / \mathrm{s})$. The $E_{1 / 2}$ values, derived from the midpoint between waves $a$ and $b$ varied linearly as function of $\mathrm{pH}$ with a slope consistent with the exchange of one proton per electron (Fig. 3 right) [40]. For wave $b^{\prime}$ the potential was stationary but its size increased with increasing $\mathrm{pH}$. Taken together, the wave $a / b$ couple (the quasi-reversible wave observed at high scan rate) was assigned to the proton coupled electron transfer of the solvated $\left[\mathrm{Mn}^{\mathrm{II}} \mathrm{L}^{\prime}\right.$ $\left.\mathrm{Gly}_{1}\left(\mathrm{H}_{2} \mathrm{O}\right)\right]^{+} /\left[\mathrm{Mn}^{\mathrm{III}} \mathrm{L}^{\prime}-\mathrm{Gly}_{1}(\mathrm{OH})\right]^{+}$couple which has been previously observed for $[\mathbf{M n L}]^{2+}[40] . \mathrm{N}$ alkylation of the ligand shifted anodically the $E_{1 / 2}$ relative to the parent compound, a behaviour which has been observed in other metal complexes containing amine ligands $[41,42]$. Wave $b^{\prime}$ was assigned to the reduction of a chemically generated product of $\left[\mathrm{Mn}^{\mathrm{III}} \mathrm{L}^{\prime}-\mathrm{Gly}(\mathrm{OH})\right]^{+}$, most likely dimeric species, which are known to be favoured at higher $\mathrm{pH}$ [43]. The CV of $\left[\mathbf{M n L}^{\prime}-\mathbf{A r g}_{1}\right]^{2+}$ and $\left[\mathbf{M n L} \mathbf{L}^{\prime}-\mathbf{A r g}_{3}\right]^{4+}$ were less reversible than $\left[\mathbf{M n L}^{\prime}-\mathbf{G l y}_{\mathbf{1}}\right]^{+}$, but behaved in a similar manner with 
varying $v$ values (Fig. 2 right, table 2). However, for derivatives with a longer peptide moiety $\left(\left[\mathbf{M n L}^{\prime}-\mathbf{A r g}_{6}\right]^{7+}\right.$ and $\left.\left[\mathbf{M n L}^{\prime}-\mathbf{A r g}_{9}\right]^{10+}\right)$ the anodic wave observed at $E_{\mathrm{p}}^{\mathrm{a}} \approx 0.35 \mathrm{~V}$ vs SCE, was followed by an ill-defined cathodic wave in the region -0.1 to $0.1 \mathrm{~V}$ vs SCE on the reverse scan. This was consistent with the slower diffusion rates of the higher molecular weight $\left[\mathbf{M n L} \mathbf{L}^{\prime}-\mathbf{R}\right]^{n+}$ species on the CV timescale such that the chemical dimerization process began to dominate and the reduction of the $\mathrm{Mn}^{\mathrm{III}}$ monomer on the reverse scan was no longer easily distinguished.

\subsection{SOD-like activity}

The SOD-like activity of $\left[\mathbf{M n L} \mathbf{L}^{\prime}-\mathbf{R}\right]^{n+}$ were screened under real catalytic conditions, with superoxide concentrations in a high excess relative to the investigated complexes. A direct stoppedflow measurement combined with fast diode-array UV-vis detection as previously described [36] was used for the quantification of the catalytic activity. The $k_{\text {cat }}$ values, corrected for the $K_{\text {assoc }}$ because of the low assaying concentration range, were determined for $\left[\mathbf{M n L}^{\prime}-\mathbf{R}\right]^{n+}$ and the parent compound at various ionic strengths $\left(\mu\right.$, table 2). Note that $\left[\mathrm{Mn}^{\mathrm{II}}\left(\mathrm{H}_{2} \mathrm{O}\right)_{\mathrm{n}}\right]^{2+}$ was expected to have no effect under these conditions [26,36]. For all compounds, the $k_{\text {cat }}$ values decreased with increasing $\mu$, consistent with the reaction between species of opposite charges. At any given $\mu\left[\mathbf{M n L}^{\prime}-\mathbf{G l y}_{\mathbf{1}}\right]^{+}$ $\left(E_{1 / 2}=0.30 \mathrm{~V}\right.$ vs SCE $)$ was less active than $[\mathrm{MnL}]^{+}\left(E_{1 / 2}=0.22 \mathrm{~V}\right.$ vs SCE $)$. This was due to the more positive $E_{1 / 2}$ value of $\left[\mathbf{M n L}^{\prime}-\mathbf{G l y}_{\mathbf{1}}\right]^{+}$being further from the optimal potential for $\mathrm{O}_{2}{ }^{-}$ dismutation.

In contrast, the $\left[\mathbf{M n L} \mathbf{L}^{\prime}-\mathbf{A r g}_{\mathbf{x}}\right]^{n+}$ derivatives, were found to be equally or more active than the parent compound at low $\mu(26 \mathrm{mM})$. This enhancement/recovery of activity could possibly be attributed to an electrostatic contribution, with an attraction between the cationic arginine side chain and the anionic substrate. Such electrostatic contributions have been previously described for MnSOD mimics, and have been found to be strongest for pentacationic porphyrinic manganese(III) complexes, where the positively charged heterocyclic nitrogen atoms were ortho to the porphyrin meso carbons, which channels $\mathrm{O}_{2}{ }^{-}$towards the active site [20,21,23]. With other factors being 
equal, the electrostatic enhancement of $k_{\text {cat }}$ values in the "ortho" porphyrins correlated directly with the number of positive charges, where by application of the Brønsted-Debye-Hückel equation the slope for $\mu^{1 / 2} /\left(1+\mu^{1 / 2}\right)$ vs. $\log \left(k_{\text {cat }}\right)$ scaled linearly with the total charge [20,21]. Weaker dependence of $k_{\text {cat }}$ on $\mu$ were observed for derivatives where the positively charged heterocyclic nitrogen atoms are meta or para to the porphyrin meso carbons [20]. This indicates that, not only the total charge, but also its topography plays an important role. Indeed, in SOD, the charge are located along a funnel leading from the protein surface to the active site [3]. In the case of the $\left[\mathbf{M n L} \mathbf{L}^{\prime}-\mathbf{A r g}_{(n-1)}\right]^{n+}$, for a given $\mu$ value the $k_{\text {cat }}$ value did not increase progressively with the number of cationic arginine residues. For the cationic MnL the slope for $\mu^{1 / 2} /\left(1+\mu^{1 / 2}\right)$ vs. $\log \left(k_{\text {cat }}\right)$ was -0.5 , while for the polycationic $\left[\mathbf{M n L}^{\prime}-\mathbf{A r g}_{(n-1)}\right]^{n+}$ the slopes were in the range of -0.9 to -1.7 (Fig. 4) which were not correlated to the number of arginine residues, suggesting that the extent of the electrostatic enhancement was similar for all $\left[\mathbf{M n L}^{\prime}-\mathbf{A r g}_{(n-1)}\right]^{n+}$. The ratio of the slopes of $\left[\mathbf{M n L}^{\prime}-\mathbf{A r g}_{(n-1)}\right]^{n+}$ to $[\mathrm{MnL}]^{+}$were between 1.8 and 3.4 , which was consistent with approximately one or two additional cationic charges being very close to the $\mathrm{Mn}$, as was the case for the four positively charged nitrogen atoms in the "ortho" porphyrins. Taken together, one possible explanation is that only the cationic guanidinium groups of the first and second arginine residue, which are in the close proximity to the Mn centre, have an electrostatic enhancement. Further elongation of the polyarginine chain has no significant effect.

\section{Conclusion}

SODs are very efficient enzymes, carved by evolution for high efficiency in the catalytic dismutation of $\mathrm{O}_{2}{ }^{-}$. Common characteristics shared by SODs of different lineage point to important physicochemical parameters that tune their efficiency, such as the redox potential and the presence of electrostatic channels for $\mathrm{O}_{2}{ }^{-}$attraction [3]. In a bio-inspired approach we have been designing Mn complexes with SOD-activity [12]. After investigating the effect of redox tuning [26], we have conjugated positively charged peptides to $[\mathbf{M n L}]^{+}$in order to vary the electrostatic 
situation close to the SOD active centre. Interestingly, we have shown that conjugation of $[\mathbf{M n L}]^{+}$ at the secondary amine of the 1,2-diaminoethane central scaffold did not impair coordination to $\mathrm{Mn}^{\mathrm{II}}$. As anticipated, the coordination of the tertiary amine was found to be enthalpically less favourable, but a more favourable entropic component led to similar association constants. The redox potential of the $\mathrm{Mn}^{\mathrm{III}} / \mathrm{Mn}^{\mathrm{II}}$ couple increases upon functionalization at the amine, but remains at a value that facilitates $\mathrm{O}_{2}{ }^{-}$dismutation. The positively charged electrostatic funnels observed in SOD have been suggested to play an important role for long-range guidance from the protein exterior towards the active site [3]. As previously shown in the case of porphyrins, the overall charge is not the only parameter but its topography $[12,22]$ and distance from the metal centre is also important. In the case of the series studied here, the analyses of the effect of the ionic strength on the $k_{\text {cat }}$ strongly suggests that not all the charges but only those that are the closest to the SOD active metal centre exerted an electrostatic favourable influence. These results are of interests for future design of SOD mimics and improvement of their catalytic activity.
Abbreviations
Boc
tert-butyloxycarbonyl
$\mathrm{CV} \quad$ cyclic voltammetry
DCM dichloromethane
DIPEA $\quad N, N$-diisopropyldthylamine
DMF $\quad N, N$-dimethylformamide
DMSO dimethylsulfoxide
ESI-Q-TOF electrospray quadrupole coupled - time of flight
Fmoc fluorenylmethyloxycarbonyl
HBTU 3-[bis(dimethylamino)methyliumyl]-3H-benzotriazol-1-oxide hexafluorophosphate
HEPES 4-(2-hydroxyethyl)piperazine-1-ethanesulfonic acid
HPLC high performance liquid chromotography
HR high resolution
Im imidazole 


\begin{tabular}{|c|c|}
\hline ITC & isothermal titration calorimetry \\
\hline MALDI-TOF & matrix-assisted laser desorption/ionisation - time-of-flight \\
\hline MBHA & 4-methylbenzhydrylamine \\
\hline $\mathrm{MeOH}$ & methanol \\
\hline $\mathrm{ACN}$ & acetonitrile \\
\hline NMP & $N$-methyl-2-pyrrolidone \\
\hline NMR & nuclear magnetic resonance \\
\hline$\phi$ & phenol \\
\hline $\mathrm{Pbf}$ & 2,2,4,6,7-pentamethyldihydrobenzofuran-5-sulfonyl \\
\hline ROS & reactive oxygen species \\
\hline SCE & standard calormel electrode \\
\hline SOD & superoxide dismutase \\
\hline TFA & trifluoroacetic acid \\
\hline TIS & triisoproplysilane \\
\hline Tos & 4-toluenesulphonyl \\
\hline
\end{tabular}

\section{Acknowledgements}

We thank Delphine Arquier and Tanya Ingeolu ((Institut de Chimie Moléculaire et des Matériaux d'Orsay, Université Paris Sud) for providing the HR-ESI-Q-TOF-MS data; Dr Sandrine Sagan (Laboratoire des Biomolécules UMR 7203, CNRS/UPMC/ENS) for support regarding the ITC experiments; Dr Cedric Tard and Dr Matteo Duca (Laboratoire d'Electrochimie Moléculaire, UMR 7591, CNRS/Université Paris Diderot - Paris 7) for help with electrochemical studies and Mr Rodrigue Marquant (Laboratoire des Biomolécules UMR 7203, CNRS/UPMC/ENS) for assistance with HPLC. ANR-Metabact and ANR-Magic (ANR-15-CE07-0027-01) are gratefully acknowledged for financial support and fellowship for H.Y.V.C.; ENS Cachan for E.M. thesis fellowship and ENS for funding her teaching. 


\section{References}

[1] P.R. Gardner, I. Raineri, L.B. Epstein, C.W. White, J. Biol. Chem. 270 (1995) 1339913405.

[2] B. Halliwell, J.M.C. Gutteridge, Free radicals in biology and medicine, 4th ed., Oxford University Press Inc., New York, 2007.

[3] Y. Sheng, I.A. Abreu, D.E. Cabelli, M.J. Maroney, A.F. Miller, M. Teixeira, J.S. Valentine, Chem. Rev. 114 (2014) 3854-3918.

[4] C. Muscoli, S. Cuzzocrea, D.P. Riley, J.L. Zweier, C. Thiemermann, Z.-Q. Wang, D. Salvemini, Br. J. Pharmacol. 140 (2003) 445-460.

[5] J.M. McCord, M.A. Edeas, Biomed. Pharmacother. 59 (2005) 139-142.

[6] I. Batinic-Haberle, A. Tovmasyan, E.R.H. Roberts, Z. Vujaskovic, K.W. Leong, I. Spasojevic, Antioxid. Redox Signal. 20 (2014) 2372-2415.

[7] I. Batinić-Haberle, J.S. Rebouças, I. Spasojević, Antioxid. Redox Signal. 13 (2010) $877-$ 918.

[8] O. Iranzo, Bioorg. Chem. 39 (2011) 73-87.

[9] S. Miriyala, I. Spasojevic, A. Tovmasyan, D. Salvemini, Z. Vujaskovic, D. St. Clair, I. Batinić-Haberle, Biochim. Biophys. Acta - Mol. Basis Dis. 1822 (2012) 794-814.

[10] D. Lieb, F.C. Friedel, M. Yawer, A. Zahl, M.M. Khusniyarov, F.W. Heinemann, I. Ivanović-Burmazović, Inorg. Chem. 52 (2013) 222-36.

[11] D. Lieb, I. Kenkell, J.L. Miljkovic, D. Moldenhauer, N. Weber, M.R. Filipovic, F. Gröhn, I. Ivanović-Burmazović, Inorg. Chem. 53 (2014) 1009-1020.

[12] C. Policar, Mimicking SOD, Why and How: Bio-Inspired Manganese Complexes as SOD Mimic, in: J.S. Reboucas, I. Batinic-Haberle, I. Spasojevic, D.S. Warner, D. St. Clair (Eds.), Redox Active Therapeutics, 2015: p. in press.

[13] W.H. Koppenol, D.M. Stanbury, P.L. Bounds, Free Radic. Biol. Med. 49 (2010) 317-322.

[14] W.C. Barrette Jr., D.T. Sawyer, J.A. Fee, K. Asada, Biochemistry. 22 (1983) 624-627.

[15] W.H. Koppenol, The physiological role of charge distribution on SOD, in: Oxygen and Oxy-Radicals in Chemistry and Biology, 1981: pp. 671-674.

[16] J. Benovic, T. Tillman, a Cudd, I. Fridovich, Arch. Biochem. Biophys. 221 (1983) 329-332.

[17] E.D. Getzoff, J. a Tainer, P.K. Weiner, P. a Kollman, J.S. Richardson, D.C. Richardson, Nature. 306 (1983) 287-290.

[18] A. Desideri, M. Falconi, V. Parisi, S. Morante, G. Rotilio, Free Radic. Biol. Med. 5 (1988) 313-317. 
[19] H.X. Zhou, K.Y. Wong, M. Vijayakumar, Proc. Natl. Acad. Sci. U. S. A. 94 (1997) 1237212377.

[20] I. Spasojević, I. Batinić-Haberle, J.S. Rebouças, Y.M. Idemori, I. Fridovich, J. Biol. Chem. 278 (2003) 6831-6837.

[21] I. Batinić-Haberle, I. Spasojević, R.D. Stevens, P. Hambright, P. Neta, A. OkadoMatsumoto, I. Fridovich, Dalton Trans. (2004) 1696-1702.

[22] J.S. Rebouças, G. DeFreitas-Silva, I. Spasojević, Y.M. Idemori, L. Benov, I. BatinićHaberle, Free Radic. Biol. Med. 45 (2008) 201-210.

[23] J.S. Rebouças, I. Spasojević, D.H. Tjahjono, A. Richaud, F. Méndez, L. Benov, I. BatinićHaberle, Dalt. Trans. (2008) 1233-1242.

[24] C. Policar, F. Lambert, M. Cesario, I. Morgenstern-Badarau, Eur. J. Inorg. Chem. (1999) 2201-2207.

[25] C. Policar, S. Durot, F. Lambert, M. Cesario, F. Ramiandrasoa, I. Morgenstern-Badarau, Eur. J. Inorg. Chem. 2001 (2001) 1807-1818.

[26] S. Durot, C. Policar, F. Cisnetti, F. Lambert, J.P. Renault, G. Pelosi, G. Blain, H. KorriYoussoufi, J.-P. Mahy, Eur. J. Inorg. Chem. (2005) 3513-3523.

[27] S. Durot, F. Lambert, J.P. Renault, C. Policar, Eur. J. Inorg. Chem. (2005) 2789-2793.

[28] F. Cisnetti, A.S. Lefèvre, R. Guillot, F. Lambert, G. Blain, E. Anxolabéhère-Mallart, C. Policar, Eur. J. Inorg. Chem. 1 (2007) 4472-4480.

[29] F. Cisnetti, G. Pelosi, C. Policar, Inorganica Chim. Acta. 360 (2007) 557-562.

[30] S. Groni, G. Blain, C. Policar, E. Anxolabéhère-Mallart, 46 (2007) 1951-1953.

[31] G.E. Borgstahl, H.E. Parge, M.J. Hickey, W.F. Beyer, R.A. Hallewell, J.A. Tainer, Cell. 71 (1992) 107-118.

[32] A.-S. Bernard, C. Giroud, H.Y.V. Ching, A. Meunier, V. Ambike, C. Amatore, M.G. Collignon, F. Lemaître, C. Policar, Dalt. Trans. 41 (2012) 6399-6403.

[33] S. Asayama, E. Kawamura, S. Nagaoka, H. Kawakami, Mol. Pharm. 3 (2006) 468-470.

[34] G. Dirscherl, B. König, Eur. J. Org. Chem. (2008) 597-634.

[35] S. Futaki, T. Suzuki, W. Ohashi, T. Yagami, S. Tanaka, K. Ueda, Y. Sugiura, J. Biol. Chem. 276 (2001) 5836-5840.

[36] F.C. Friedel, D. Lieb, I. Ivanović-Burmazović, J. Inorg. Biochem. 109 (2012) 26-32.

[37] L.A.K. Staveley, T. Randall, Discuss. Faraday Soc. 26 (1958) 157-163.

[38] A. Bencini, A. Bianchi, P. Dapporto, E. Garcia-Espana, V. Marcelino, M. Micheloni, P. Paoletti, P. Paoli, Inorg. Chem. 29 (1990) 1716-1718. 
[39] A. Bianchi, L. Calabi, C. Giorgi, P. Losi, P. Mariani, D. Palano, P. Paoli, P. Rossia, B., J. Chem. Soc. Dalt. Trans. (2001) 917-922.

[40] E. Anxolabéhère-Mallart, C. Costentin, C. Policar, M. Robert, J.-M. Savéant, A.-L. Teillout, Faraday Discuss. 148 (2011) 83-95; discussion 97-108.

[41] D. Meyerstein, Coord. Chem. Rev. 185-186 (1999) 141-147.

[42] E.K. Barefield, Coord. Chem. Rev. 254 (2010) 1607-1627.

[43] M.N. Collomb, A. Deronzier, Eur. J. Inorg. Chem. (2009) 2025-2046. 

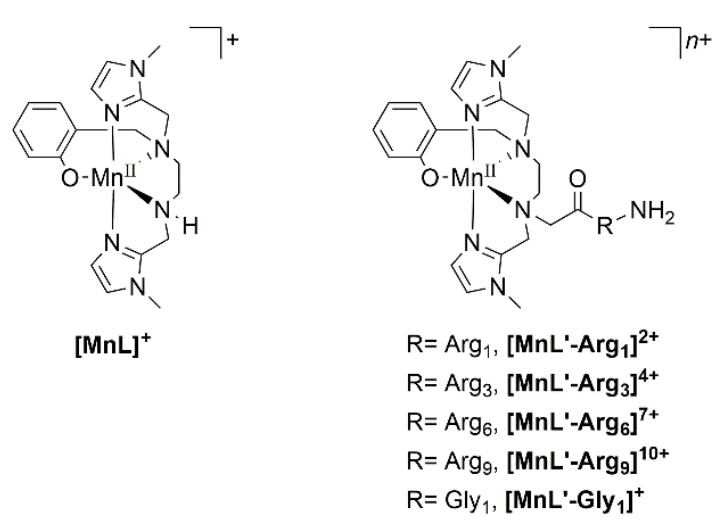

Scheme 1. $[\mathbf{M n L}]^{+}$and peptidic derivatives $[\mathbf{M n L}-\mathbf{R}]^{n+}$
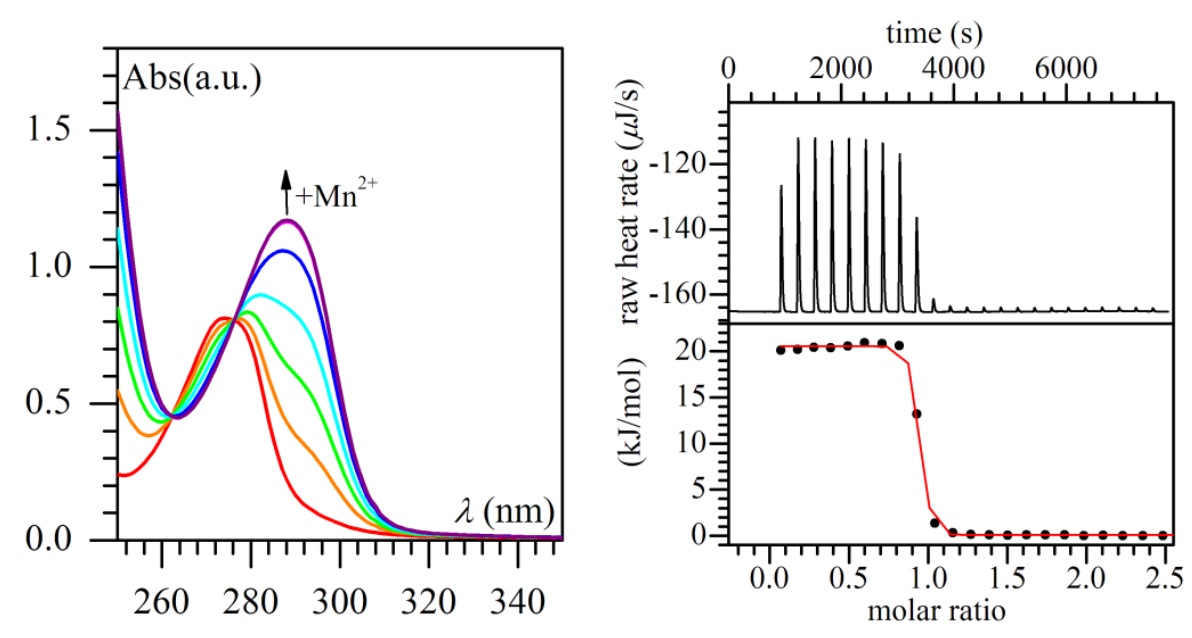

Fig. 1. left panel: Selected UV-vis spectrum of HL-Arg $(0.55 \mathrm{mM})$ with increasing concentration of $\mathrm{MnCl}_{2}$ (0 to $\left.0.70 \mathrm{mM}\right)$ in HEPES $(100 \mathrm{mM}, \mathrm{pH} 7.5)$. right panel: ITC profile for the complexation of $\mathrm{Mn}^{\mathrm{II}}$ ion to $\mathrm{HL}^{\prime}$ - $\mathbf{A r g}_{\mathbf{6}}$ in HEPES $(100 \mathrm{mM}, \mathrm{pH} 7.5)$ at $25^{\circ} \mathrm{C}$. The top panel shows the power $(\mu \mathrm{J} / \mathrm{s})$ that needed to be applied to the sample cells to maintain isothermal conditions with respect to the reference cells. In the bottom panel the heat evolved from each injection per mole of compound (obtained from integrating the individual heat pulses of the upper panels) versus the molar ratio is presented. The solid lines in the lower panel represent a fit of one-site binding models to the data. 
Table 1. Thermodynamic parameters for complexation of $\mathrm{Mn}^{\mathrm{II}}$ to $\mathbf{H L}$ and $\mathbf{H L}$ '-R in HEPES (100 $\left.\mathrm{mM}, \mathrm{pH} 7.5,25^{\circ} \mathrm{C}\right)$ as determined by ITC.

\begin{tabular}{|c|c|c|c|c|c|c|}
\hline & $N$ & $K_{\text {assoc }} / 10^{6} \mathrm{M}^{-1}$ & $K_{\mathrm{d}} / 10^{-7} \mathrm{M}$ & $\Delta H / \mathrm{kJ} / \mathrm{mol}$ & $\Delta S / \mathrm{J} / \mathrm{mol} / \mathrm{K}$ & $\Delta G / \mathrm{kJ} / \mathrm{mol}$ \\
\hline HL & $0.9 \pm 0.2$ & $1.6 \pm 0.3$ & $6.5 \pm 1.7$ & $2.6 \pm 0.2$ & $124 \pm 1$ & $-34.5 \pm 0.1$ \\
\hline $\mathrm{HL}^{\prime}-\mathrm{Arg}_{1}$ & $1.2 \pm 0.1$ & $3.3 \pm 0.2$ & $3.0 \pm 0.2$ & $13.4 \pm 0.1$ & $170 \pm 1$ & $-37.2 \pm 0.2$ \\
\hline HL'$^{\prime}-\mathrm{Arg}_{3}$ & $0.9 \pm 0.1$ & $2.7 \pm 0.1$ & $3.7 \pm 0.1$ & $17.3 \pm 0.1$ & $181 \pm 1$ & $-36.7 \pm 0.1$ \\
\hline HL'- $\operatorname{Arg}_{6}$ & $0.9 \pm 0.1$ & $2.2 \pm 0.1$ & $4.5 \pm 0.2$ & $20.0 \pm 0.1$ & $188 \pm 1$ & $-36.2 \pm 0.1$ \\
\hline HL'- Arg 9 & $1.2 \pm 0.1$ & $1.6 \pm 0.1$ & $6.3 \pm 0.5$ & $16.3 \pm 0.3$ & $174 \pm 1$ & $-35.4 \pm 0.2$ \\
\hline HL'-Gly & $0.9 \pm 0.1$ & $3.3 \pm 0.3$ & $3.1 \pm 0.3$ & $9.9 \pm 0.1$ & $157 \pm 1$ & $-37.2 \pm 0.3$ \\
\hline
\end{tabular}
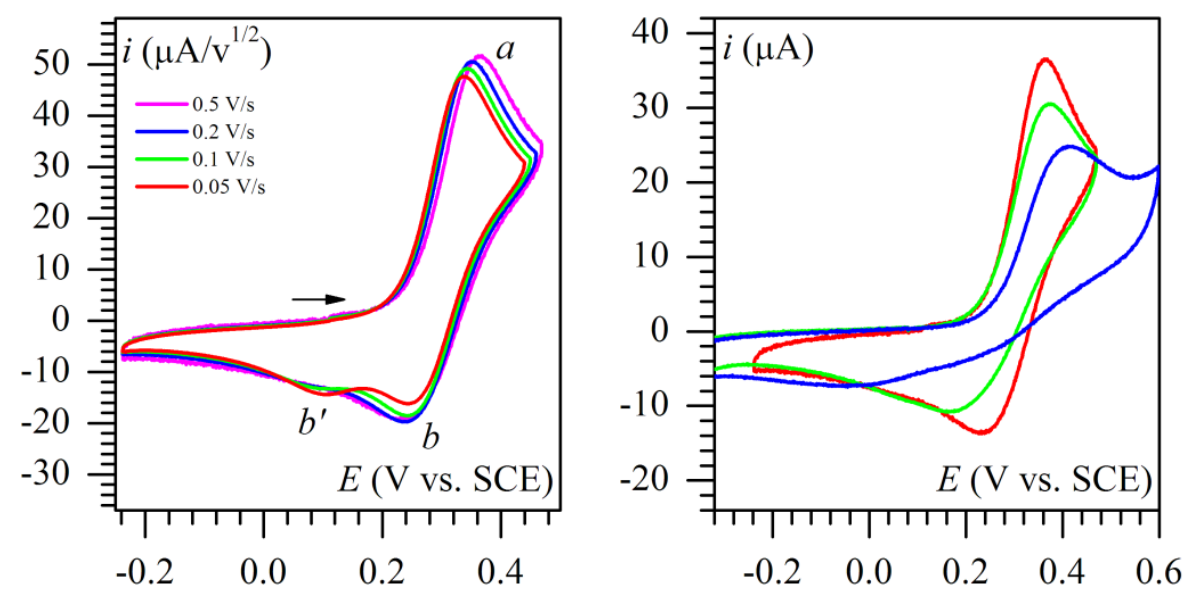

Fig. 2. left: Normalised CV of [MnL'-Gly $]^{+}(1.0 \mathrm{mM})$ in HEPES (100 mM, pH 7.5, RT) at a glassy carbon disk electrode at varying scan rates (v): 0.05 (red), 0.1 (green), 0.2 (blue), and 0.5 (magenta) V/s. right $\mathbf{C V}$ of selected $\left[\mathbf{M n L} \mathbf{L}^{\prime}-\mathbf{G l y}\right]^{+}$(red), $\left[\mathbf{M n L}-\mathbf{A r g}_{3}\right]^{4+}$ (green), and $\left[\mathbf{M n L}^{\prime}-\mathbf{A r g}_{6}\right]^{7+}$ (blue) $(1.0 \mathrm{mM})$ in HEPES $(100 \mathrm{mM}, \mathrm{pH} 7.5, \mathrm{RT})$ at a glassy carbon disk electrode at $v=0.5 \mathrm{~V} / \mathrm{s}$. 

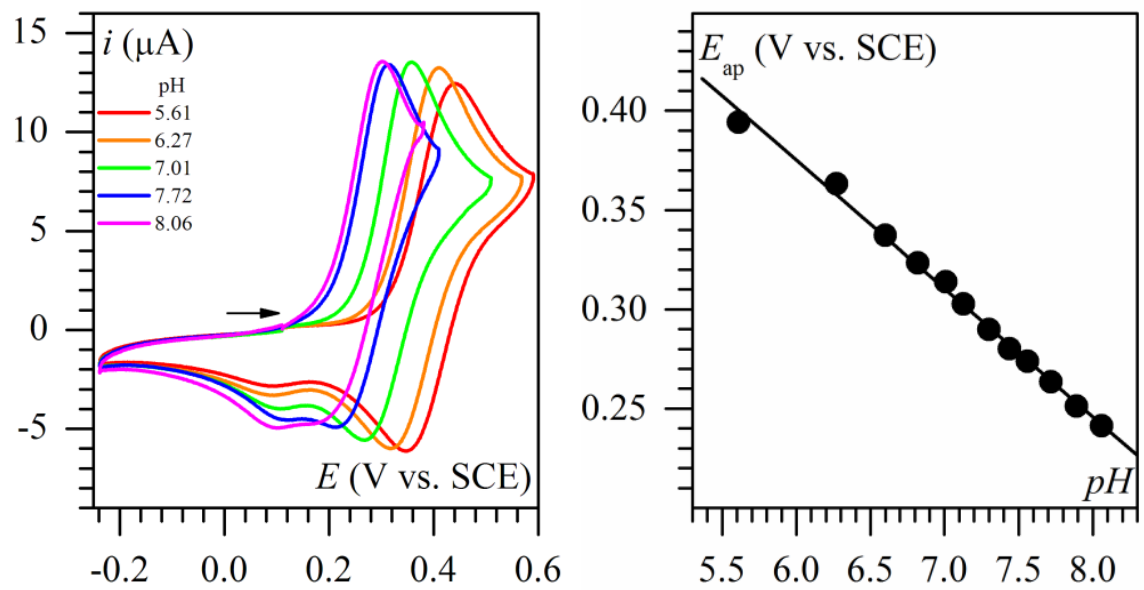

Fig. 3. left Selected CV of [MnL'-Gly $]^{+}(1.0 \mathrm{mM})$ in water (RT) with HEPES (100 mM) and $\mathrm{NaCl}$ $(100 \mathrm{mM})$ at a glassy carbon disk electrode at $v=0.1 \mathrm{~V} / \mathrm{s}$ at varying $\mathrm{pH}$ : 5.61 (red), 6.27 (orange), 7.01 (green), 7.72 (blue), and 8.06 (magenta). right: Plot of $E_{1 / 2}$ vs $\mathrm{pH}$.

Table 2. $k_{\text {cat }}$ values at different ionic strengths (60 mM HEPES, pH 7.4, with 0, 86, 186 and 286 $\mathrm{mM} \mathrm{NaCl}$ ) determined by stopped-flow technique combined with fast diode-array UV-vis; $E_{1 / 2}$ in HEPES $\left(100 \mathrm{mM}, \mathrm{pH} 7.5,25^{\circ} \mathrm{C}\right)$ determined by cyclic voltammetry for $[\mathbf{M n L}]^{+}$and $\left[\mathbf{M n L} \mathbf{L}^{\prime}-\mathbf{R}\right]^{n+}$.

\begin{tabular}{|c|c|c|c|c|c|c|}
\hline & & \multicolumn{4}{|c|}{$k_{\text {cat }}\left(10^{6} \mathrm{M}^{-1} \mathrm{~s}^{-1}\right)$} & \multirow[t]{2}{*}{$E_{1 / 2}(\mathrm{~V} v s . \mathrm{SCE})$} \\
\hline & $\mu(\mathrm{mM})$ & 26 & 112 & 212 & 312 & \\
\hline$\left[\mathrm{MnL}^{+}\right.$ & & $5.0 \pm 0.1$ & $\mathrm{n} / \mathrm{a}$ & $4.3 \pm 0.1$ & $3.7 \pm 0.1$ & 0.22 \\
\hline$\left[\mathrm{MnL}^{\prime}-\mathrm{Arg}_{1}\right]^{+}$ & & $6.6 \pm 0.1$ & $6.2 \pm 0.1$ & $4.5 \pm 0.1$ & $4.4 \pm 0.1$ & 0.27 \\
\hline$\left[\mathrm{MnL}^{\prime}-\mathrm{Arg}_{3}\right]^{4+}$ & & $5.1 \pm 0.3$ & $4.0 \pm 0.2$ & $3.7 \pm 0.5$ & $3.0 \pm 0.2$ & 0.27 \\
\hline$\left[\mathrm{MnL}^{\prime}-\operatorname{Arg}_{6}\right]^{7+}$ & & $6.6 \pm 0.3$ & $4.0 \pm 0.2$ & $3.6 \pm 0.2$ & $2.7 \pm 0.1$ & $\mathrm{n} / \mathrm{a}$ \\
\hline$\left[\mathrm{MnL}^{\prime}-\mathrm{Arg}_{9}\right]^{10+}$ & & $5.0 \pm 0.4$ & $4.3 \pm 0.2$ & $3.3 \pm 0.1$ & $3.2 \pm 0.1$ & $\mathrm{n} / \mathrm{a}$ \\
\hline$\left[\mathbf{M n L}^{\prime}-\mathbf{G l y}_{1}\right]^{+}$ & & $4.2 \pm 0.1$ & $2.9 \pm 0.1$ & $2.5 \pm 0.1$ & $2.4 \pm 0.1$ & 0.30 \\
\hline
\end{tabular}




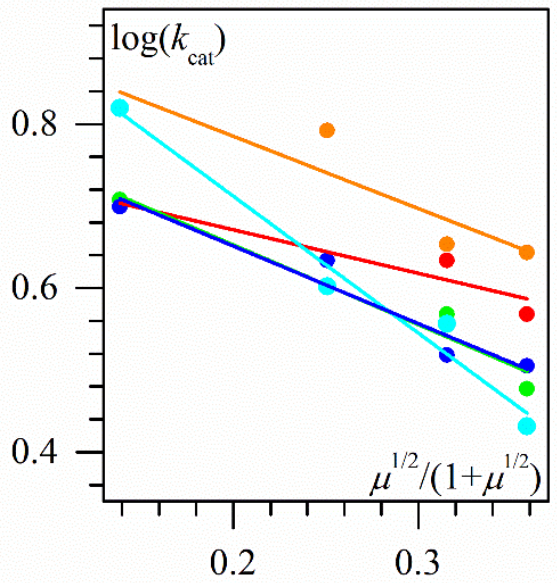

Fig. 4. $\log \left(k_{\text {cat }}\right)$ versus $\mu^{1 / 2} /\left(1+\mu^{1 / 2}\right)$ for $[\mathbf{M n L}]^{+}$(red) and $\left[\mathbf{M n L} \mathbf{L}^{\prime}-\mathbf{A r g}\right]^{2+}$ (orange), $\left[\mathbf{M n L} \mathbf{L}^{\prime}-\mathbf{A r g}_{3}\right]^{4+}$ (green), $\left[\mathbf{M n L}^{\prime}-\mathbf{A r g}_{6}\right]^{7+}$ (cyan) and $\left[\mathbf{M n L}^{\prime}-\mathbf{A r g}_{9}\right]^{10+}$ (blue). Slopes of the linear fits (solid lines) were $-0.5,-0.9,-1.0,-1.7$ and -1.0 , respectively. 